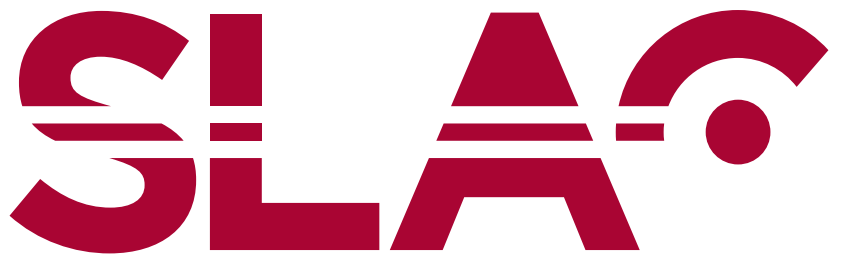

\title{
A proof-of-principle echo-enabled harmonic generation free electron laser experiment at SLAC
}

\author{
Pierre-Louis Pernet \\ École Polytechnique Fédérale de Lausanne, Lausanne, Switzerland \\ Supervised by: \\ Prof. Leonid Rivkin \\ Paul Scherrer Institut, Villigen, Switzerland \\ Prof. Tor Raubenheimer \\ SLAC National Accelerator Laboratory, Menlo Park, USA
}

June 24, 2010

\begin{abstract}
With the advent of X-ray Free Electron Lasers (FELs), new methods have been developed to extend capabilities at short wavelengths beyond Self-Amplified Spontaneous Emission (SASE). In particular, seeding of a FEL allows for temporal control of the radiation pulse and increases the peak brightness by orders of magnitude. Most recently, Gennady Stupakov and colleagues at SLAC proposed a new technique: Echo-Enabled Harmonic Generation (EEHG). Here a laser microbunches the beam in an undulator and the beam is sheared in a chicane. This process is repeated with a second laser, undulator and chicane. The interplay between these allows a seeding of the X-ray laser up to the 100th harmonic of the first laser. After introducing the physics of FELs and the EEHG seeding technique, we describe contributions to the experimental effort. We will present detailed studies of the experiment including the choice of parameters and their optimization, the emittance effect, spontaneous emission in the undulators, the second laser phase effect, and measurements of the jitter between RF stations. Finally, the status and preliminary results of the Echo-7 experiment will be outlined.
\end{abstract}




\section{Contents}

1 Introduction $\quad 3$

1.1 History of synchrotron radiation . . . . . . . . . . . . . 3

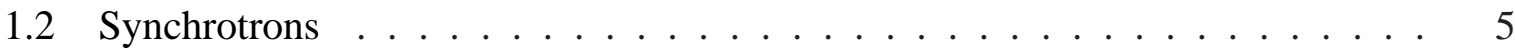

1.3 Free Electron Lasers _. . . . . . . . . . . . . . . . . . . . . 7

1.4 Linac Coherent Light Source . . . . . . . . . . . . . . . . . . . . . . 13

1.5 Seeding techniques for FELs . . . . . . . . . . . . . . . . . . . 14

2 Theoretical basis of the EEHG concept 15

2.1 Energy modulation in the first undulator . . . . . . . . . . . . . 15

2.2 Beam dispersion in a chicane section . . . . . . . . . . . . . 17

2.3 Density modulation after the first modulation section . . . . . . . . . . . 19

3 The Echo-7 experiment $\quad 21$

3.1 Choice of the parameters . . . . . . . . . . . . . . . 21

3.2 Optimization of the parameters for the two chicanes . . . . . . . . . 26

3.3 Smearing effect due to the emittance . . . . . . . . . . 30

3.4 Spontaneous emission in the undulators . . . . . . . . . . . . . 33

3.5 Second laser phase effect . . . . . . . . . . . . . . 37

3.6 Measurement of the jitter in the RF structures . . . . . . . . . . . . . . 41

4 Status of the Echo-7 experiment $\quad 45$

4.1 Current results obtained . . . . . . . . . . . . . . . . 45

4.2 A possible outlook: Echo-15 . . . . . . . . . . . . . . 46

5 Conclusion $\quad 48$

$6 \quad$ Acknowledgments $\quad 49$

A Electron-laser interaction $\quad \mathbf{5 0}$

B Echo-7 beamline $\quad 53$ 


\section{Introduction}

\subsection{History of synchrotron radiation}

In science and especially in physics, fundamental theories can still have new applications more than one century after their discovery. A famous example are the classical laws of electromagnetism, which were synthesized by J. C. Maxwell in 1873. These have enabled light source facilities as we know now, thus revolutionizing many other fields of science such as medicine, solid state physics, biology, over the past decades.

These developments started from the four Maxwell's equations described by Eqs. (1), (2), (3), and (4), with $E$ the electric field, $B$ the magnetic field, $\rho$ the charge density, and $J$ the vector current density. Maxwell predicted that a change of charge density and electric currents would create radiation [1].

$$
\begin{aligned}
\nabla \cdot E & =4 \pi \rho \\
\nabla \times E & =-\frac{1}{c} \frac{\partial B}{\partial t}, \\
\nabla \cdot B & =0 \\
\nabla \times B & =\frac{4 \pi}{c} J+\frac{1}{c} \frac{\partial E}{\partial t} .
\end{aligned}
$$

Five years later in 1887, H. Hertz demonstrated the existence of this radiation by measuring its wave properties [2]. This was followed by the discovery of retarded potentials, also known as the Liénard-Wiechert potentials. In 1898, the French scientist A. Liénard was the first to study the electric and magnetic fields produced by an electric point charge moving on an arbitrary path [3]. His work has been complemented by E. Wiechert in 1900, who studied seismic waves [4].

Finally, these major breakthroughs led to the work of G. A. Schott on electromagnetic radiation, describing, among other examples, the case of radiation coming from the energy losses of an electron traveling in a circular orbit [5]. This process is now called synchrotron radiation.

Experimentalists, working together with theoreticians, led to the construction of a new machine: the betatron. In 1940, D. Kerst completed the first one at the University of Illinois. One year later, the General Electric Research Laboratory interested in this technology, hired him to build a new one. He was assisted by W. F. Westendorp. This betatron was able to accelerate electrons up to an energy of $20 \mathrm{MeV}$.

Over the next five years, physicists and engineers managed to improve the energy of betatrons by a factor of five. In 1946, J. P. Blewett's betatron reached an energy of $100 \mathrm{MeV}$. Nevertheless, due to an opaque vacuum chamber, physicists were not able to measure visible synchrotron radiation. 
The betatron, being technically limited in energy, was surpassed by the synchrotron, invented by E. McMillan and V. Veksler in 1945. This new accelerator allowed detection of synchrotron light in the optical wavelength range. The first observation was made with the $70 \mathrm{MeV}$ General Electric synchrotron in 1947 (Fig. 1). One can see synchrotron light coming out from the chamber.

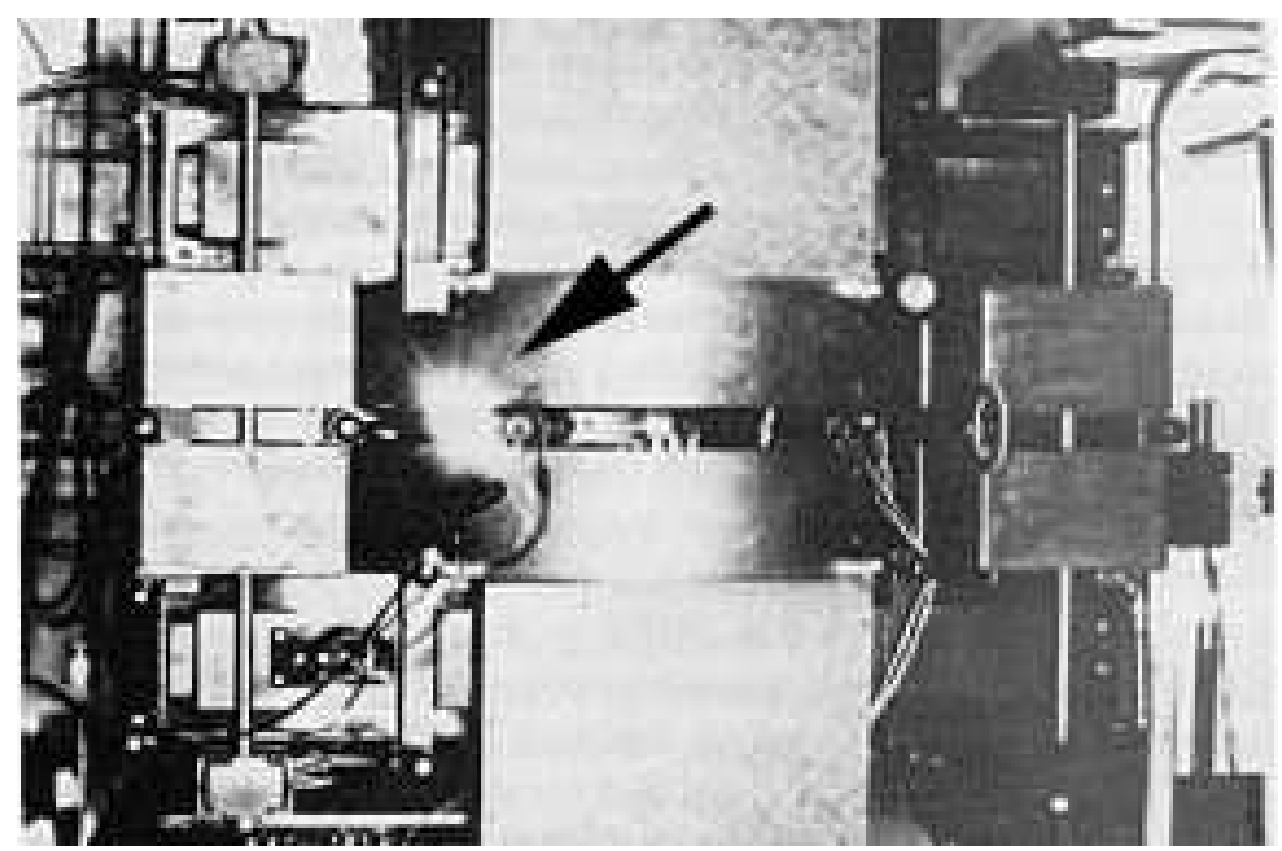

Figure 1: Synchrotron light at GE Research Laboratory [6]. 


\subsection{Synchrotrons}

During the last 50 years, the number of synchrotrons has greatly increased to about 60 ring sources on almost every continent. Another measure of success are the 60000 facility users coming from all different disciplines. The first generation of light sources we previously described, was not only dedicated for synchrotron radiation, but also for other high energy physics programs. New facilities have since been built with the sole purpose to produce synchrotron radiation used by other experiments. This second generation of synchrotrons was not designed for low emittance. Finally, third generation facilities optimized for low emittance replaced the previous ones. One representative of the third generation is the Swiss Light Source located at the Paul Scherrer Institut in Zürich (Switzerland). It was commissioned in 2001 after ten years of research and construction. An aerial photograph is presented in Fig. 2. One can see the $2.4 \mathrm{GeV}$ storage ring at the bottom right of the picture.

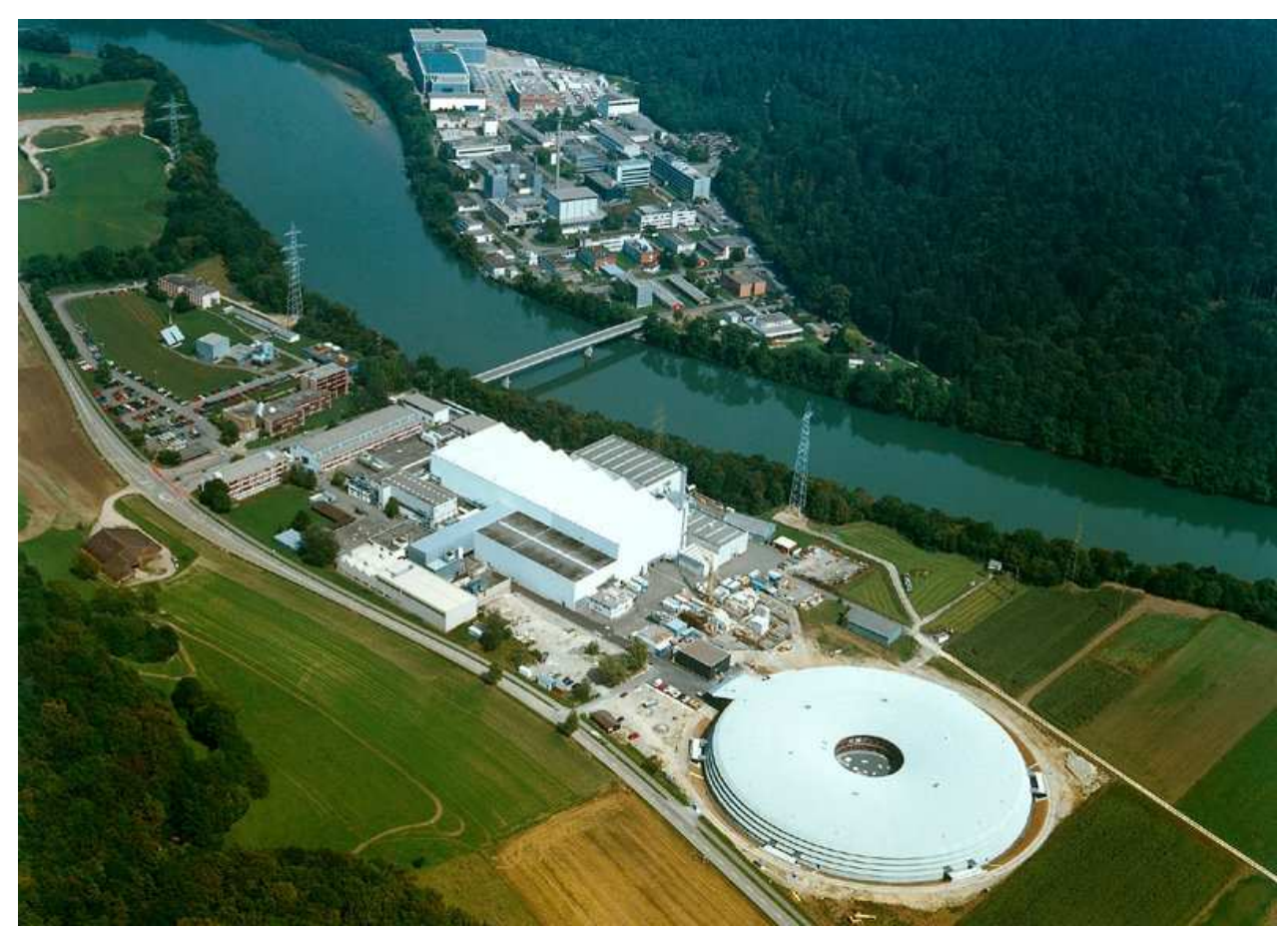

Figure 2: The Swiss Light Source at the Paul Scherrer Institut [7].

The effectiveness of a synchrotron is defined by its brightness. Brightness is proportional to the ratio between the flux and the source area times the angular divergence. Since E. McMillan and V. Veksler's synchrotron, it has been considerably increased by twelve orders of magnitude. A major consequence of this improvement is the increase of the number of photons which can be focused on a small sample. As a result one can analyze smaller objects down to the submicrometer, a human cell being between $4 \mu \mathrm{m}$ and $130 \mu \mathrm{m}$. 
The light produced by synchrotrons has a multitude of advanced applications for medicine, solid state physics, biology and others. One of the best known ones is protein crystallography $^{1)}$. It consists of illuminating a sample with X-rays in order to deduce its structure from its diffraction patterns. Synchrotron radiation is also useful for medical imaging. For example, micro-tomography, which can be performed with synchrotron X-rays, is studying the brain blood vessels in three dimensions in order to understand Alzheimer disease. Another application is X-ray phase contrast imaging. This technique is used to study the skeletons of small dead animals, such as spiders, dust mites, with a better resolution compared to conventional X-ray radiography [8]. The comparison between these two methods for a fish is presented in Fig. 3.
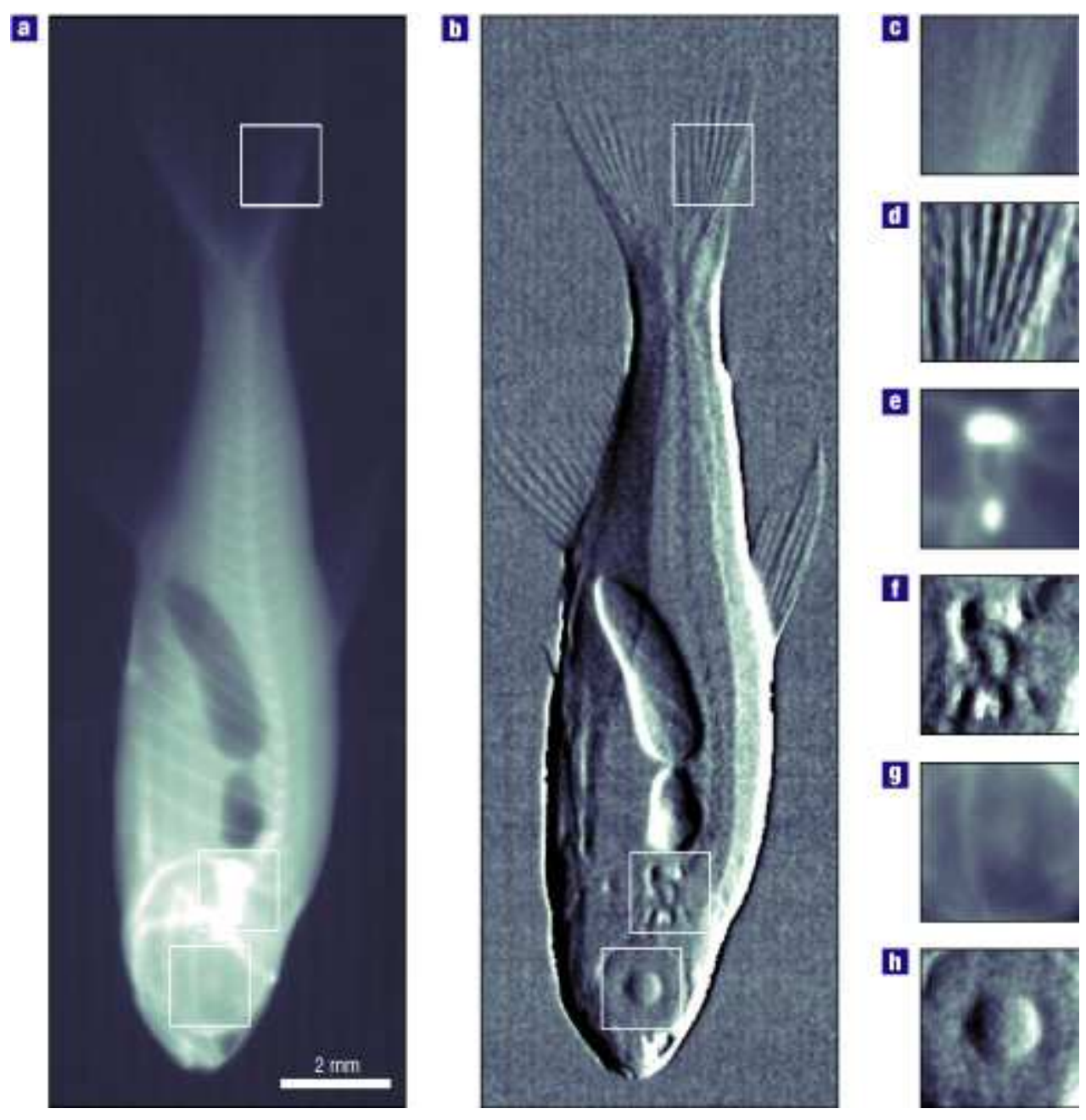

Figure 3: Comparison between the conventional X-ray transmission image of a fish a), and the differential phase-contrast image for the same fish b). Details are presented in c) to h) [9].

1) Protein crystallography has led to three Nobel Prizes since 1997 . The first one obtained by J. E. Walker for the structure of F1-ATPase. The second one has been attributed in 2003 to R. McKinnon for his discovery of the structure of cellular ion channels. Finally, R. D. Kronberg received the third one four years ago for the structure of the RNA polymerase [8] 


\subsection{Free Electron Lasers}

The next step to third generation is providing light sources with extreme brightness. One of the goals is to time resolve chemical reactions, like complex dynamical changes of large groups of atoms in disordered materials such as polymer diffusion, crystalline phase transitions. Stanford University has a great tradition to time resolve physical processes. In $1878 \mathrm{E}$. Muybridge tracked motion of an horse by recording a movie on the actual campus. A good century later, a new light source has been commissioned here again to study the evolution of a molecule during a Coulomb explosion. This new generation of light sources has to move from rings to linear accelerators, thus a new machine has been developed: the Free Electron Laser (FEL), pioneered by J. Madey in 1976 at Stanford University. The first FEL in operation in the hard $\mathrm{X}$-ray regime ${ }^{2)}$ is the LCLS commissioned last year at SLAC.

The basic principle of a FEL is the interaction between a beam of relativistic electrons moving through an undulator or wiggler, and the light they produce. An undulator or wiggler consists of a periodic sequence of dipole magnets with alternating poles (Fig. 4). The difference between an undulator and a wiggler is the size of the undulations compared to the opening angle of the radiation, small for an undulator, large for a wiggler.

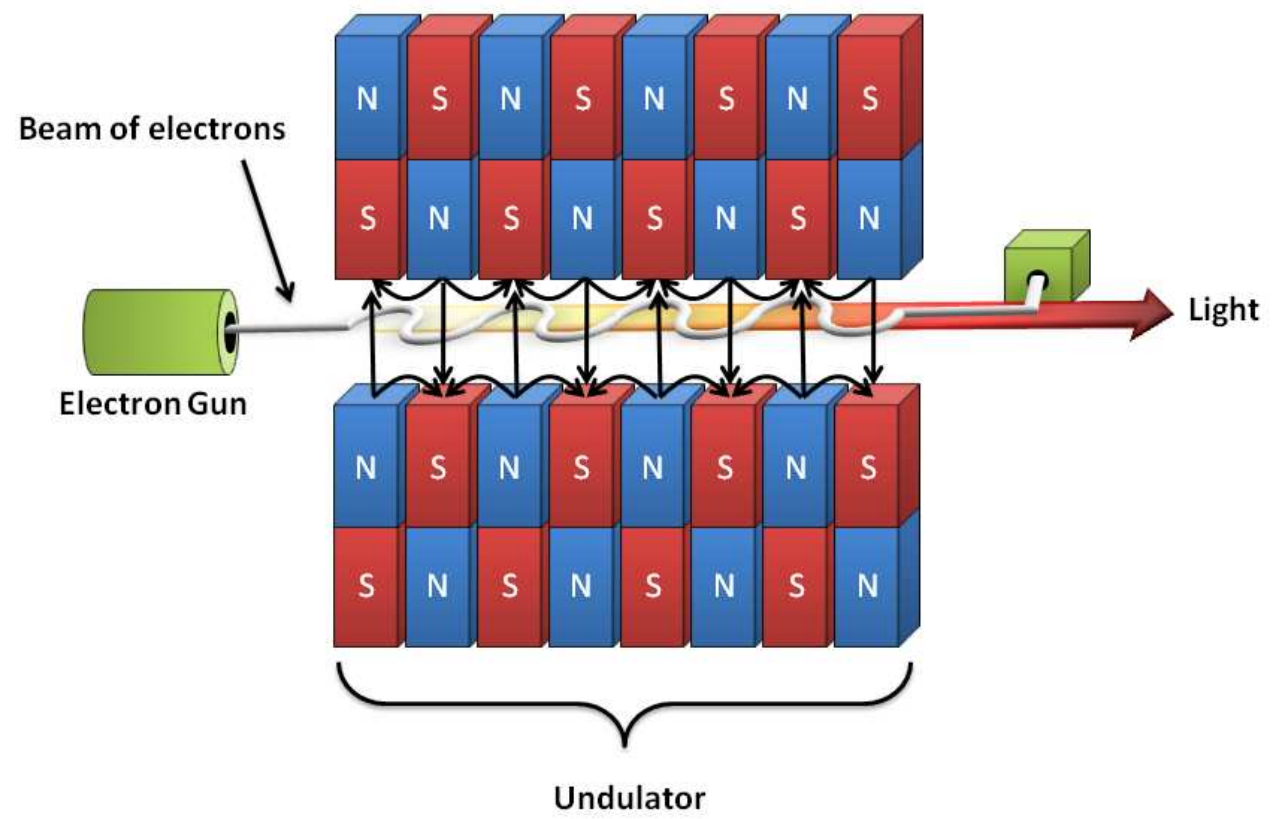

Figure 4: Basic schema of a Free Electron Laser.

2) X-rays are electromagnetic radiation with a wavelength between $0.01 \mathrm{~nm}$ and $10 \mathrm{~nm}$. They are split into two categories. Soft X-rays have a wavelength between $0.1 \mathrm{~nm}$ and $10 \mathrm{~nm}$; hard X-rays between $0.01 \mathrm{~nm}$ and $0.1 \mathrm{~nm}$. 
The light produced at the end of the undulator has a fixed resonant wavelength we will derive now. With $s$ being the flight direction of an electron, and $x$ and $y$ its transverse displacements respectively in the horizontal and vertical planes (Fig 5), the trajectory of an electron in an undulator or a wiggler, is described by the Lorentz force laws:

$$
\begin{aligned}
& \ddot{x}=\frac{d^{2} x}{d s^{2}}=\frac{e}{\gamma m_{0} c}\left(B_{y}-\dot{y} B_{s}\right), \\
& \ddot{y}=\frac{d^{2} y}{d s^{2}}=\frac{e}{\gamma m_{0} c}\left(\dot{x} B_{s}-B_{x}\right) .
\end{aligned}
$$

The assumption of small angular deflections has been made, i.e. $\dot{x} \ll 1$ and $\dot{y} \ll 1$. The symbol $e$ corresponds to the elementary electrical charge, $m_{0}$ is the rest mass of the electron, $\gamma$ the Lorentz factor, $B_{x}, B_{y}$ and $B_{s}$ represent the three components of the magnetic field at the location of the electron. In general, a planar undulator only deflects the electrons in one plane. Let's assume in the horizontal plane, therefore $B_{x}=B_{s}=0$. Eq. (5) is reduced to:

$$
\begin{aligned}
\ddot{x} & =\frac{e B_{y}}{\gamma m_{0} c}, \\
\ddot{y} & =0 .
\end{aligned}
$$

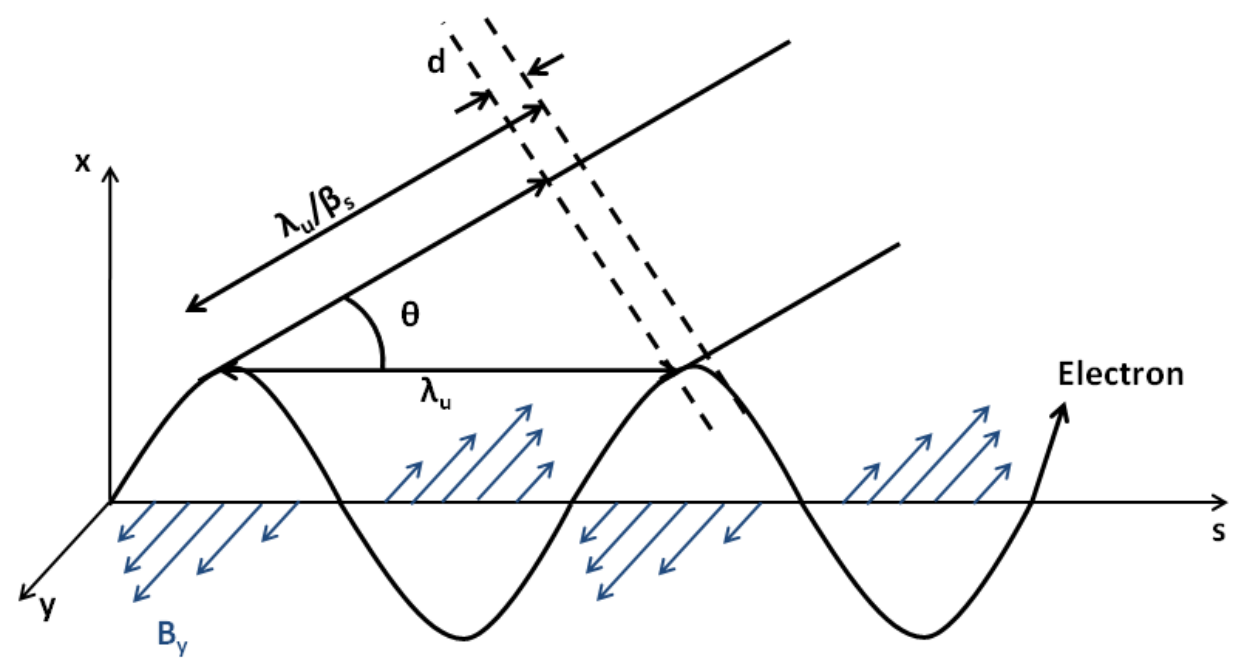

Figure 5: Trajectory of an electron in the undulator.

For $x=0$, the vertical magnetic field of the undulator can be considered sinusoidal with:

$$
B_{y}(s)=-B_{0} \sin \left(\frac{2 \pi s}{\lambda_{u}}\right)
$$

where $\lambda_{u}$ is the undulator period, and $B_{0}$ the magnetic peak field. The horizontal deflection 
angle $\dot{x}$ is determined by integrating Eq. (6):

$$
\begin{aligned}
\dot{x}(s) & =-\int \frac{B_{0} e}{\gamma m_{0} c} \sin \left(\frac{2 \pi s}{\lambda_{u}}\right) d s \\
& =\frac{B_{0} e}{\gamma m_{0} c} \frac{\lambda_{u}}{2 \pi} \cos \left(\frac{2 \pi s}{\lambda_{u}}\right) .
\end{aligned}
$$

The deflection parameter $K$, which is proportional to the maximum deflection angle of an electron is defined by:

$$
K=\frac{B_{0} e}{m_{0} c} \frac{\lambda_{u}}{2 \pi}
$$

Typically for an undulator, $K<1$, whereas for a wiggler $K \gg 1$. Finally, Eq. (8) can be rewritten as:

$$
\dot{x}(s)=\frac{K}{\gamma} \cos \left(\frac{2 \pi s}{\lambda_{u}}\right) .
$$

Using the approximation that $\Delta s=c \Delta t$ for small displacements, one has

$$
\frac{\Delta x}{\Delta s}=\frac{\Delta x}{c \Delta t}=\frac{1}{c} \frac{d x}{d t}
$$

The relative transverse velocity $\beta_{x}$ is given by:

$$
\begin{aligned}
\beta_{x} & =\frac{d x / d t}{c} \\
& =\frac{K}{\gamma} \cos \left(\frac{2 \pi s}{\lambda_{u}}\right) .
\end{aligned}
$$

With $\beta^{2}=\beta_{x}^{2}+\beta_{s}^{2}$, and approximating that the energy of the electron is constant, the relative longitudinal velocity can be expressed as:

$$
\begin{aligned}
\beta_{s}^{2} & =\beta^{2}-\beta_{x}^{2} \\
& =\beta^{2}-\frac{K^{2}}{\gamma^{2}} \cos ^{2}\left(\frac{2 \pi s}{\lambda_{u}}\right) \\
& =\beta^{2}-\frac{K^{2}}{\gamma^{2}}\left(\frac{1}{2}+\frac{1}{2} \cos \left(\frac{4 \pi s}{\lambda_{u}}\right)\right) \\
& =\beta^{2}\left[1-\frac{K^{2}}{\beta^{2} \gamma^{2}}\left(\frac{1}{2}+\frac{1}{2} \cos \left(\frac{4 \pi s}{\lambda_{u}}\right)\right)\right] .
\end{aligned}
$$

The trigonometric identity $\cos (2 \varphi)=2 \cos ^{2}(\varphi)-1$ has been applied in Eq. (13c). Performing the Taylor expansion:

$$
(1-x)^{a} \approx 1-a x
$$

Eq. (13d) can be approximated as:

$$
\beta_{s} \approx \beta\left(1-\frac{K^{2}}{4 \beta^{2} \gamma^{2}}-\frac{K^{2}}{4 \beta^{2} \gamma^{2}} \cos \left(\frac{4 \pi s}{\lambda_{u}}\right)\right)
$$


Averaging the relative longitudinal velocity over $s$ results in:

$$
\begin{aligned}
\hat{\beta}_{s} & \approx \beta-\frac{K^{2}}{4 \beta \gamma^{2}} \\
& \approx 1-\frac{1}{2 \gamma^{2}}-\frac{K^{2}}{4 \beta \gamma^{2}}
\end{aligned}
$$

The magnetic field produced by the undulator forces the electrons to follow a sinusoidal path. They emit synchrotron radiation around their direction of motion, by transferring a part of their energy to a co-propagating electromagnetic wave. In order to obtain some light at the end of the undulator, constructive interference needs to occur between all the wave-fronts emitted by one electron (Fig 5). The distance $d$ between two wave-fronts emitted with an angle $\theta$ is:

$$
d=\frac{\lambda_{u}}{\hat{\beta}_{s}}-\lambda_{u} \cos \theta
$$

An electron travels one full period of the undulator in $T=\lambda_{u} / c \hat{\beta}_{s}$. During this time $T$, the first wavefront of the co-propagating electromagnetic wave has traveled $\lambda_{u} / \hat{\beta}_{s}$. One condition required for constructive interference, is that the distance between the first wavefront and the electron has to be equal to a whole number of wavelengths over one period:

$$
n \lambda=\frac{\lambda_{u}}{\hat{\beta}_{s}}-\lambda_{u} \cos \theta
$$

Using Eqs. (14) and (16), the condition for constructive interferences is now:

$$
\begin{aligned}
n \lambda & \approx \lambda_{u}\left(1+\frac{1}{2 \gamma^{2}}+\frac{K^{2}}{4 \beta \gamma^{2}}\right)-\lambda_{u} \cos \theta \\
& \approx \lambda_{u}(1-\cos \theta)+\frac{\lambda_{u}}{2 \gamma^{2}}+\frac{\lambda_{u} K^{2}}{4 \beta \gamma^{2}} \\
& \approx \frac{\lambda_{u} \theta^{2}}{2}+\frac{\lambda_{u}}{2 \gamma^{2}}+\frac{\lambda_{u} K^{2}}{4 \beta \gamma^{2}} \\
& \approx \frac{\lambda_{u}}{2 \gamma^{2}}\left(1+\frac{K^{2}}{2}+\theta^{2} \gamma^{2}\right)
\end{aligned}
$$

where the trigonometric identity $1-\cos (\varphi)=2 \sin ^{2}(\varphi / 2)$ was used in Eq. (19c). The assumption of small angles was made, i.e. $\sin (\varphi) \approx \varphi$. Eventually, the resonant wavelength $\lambda$ is given by:

$$
\lambda=\frac{\lambda_{u}}{2 n \gamma^{2}}\left(1+\frac{K^{2}}{2}+\theta^{2} \gamma^{2}\right) .
$$

One major consequence of Eq. (20) is the possibility of continuously tuning the light produced by a FEL. The resonance wavelength $\lambda$ depends on the deflection parameter $K$, which is proportional to the magnetic field $B_{0}$ of the undulator. This field can be easily adjusted in undulators by increasing or decreasing the gap between magnets. Moreover, the wavelength $\lambda$ changes to the observation angle $\theta$, as shown below. 
In a FEL, the combination of the field produced by the undulator and the co-propagating electromagnetic wave self-bunches the beam. This resulting wave has a frequency $\omega$ and a wavenumber $k+k_{u}$, with $k=2 \pi / \lambda$ and $k_{u}=2 \pi / \lambda_{u}$. Its phase velocity is given by $\omega /\left(k+k_{u}\right)$, which is less than the speed of light. The phase $\psi$ of this wave is given by [10]:

$$
\psi=\left(k+k_{u}\right) z-\omega \bar{t}
$$

where $\bar{t}$ is the average arrival time of the electrons. The evolution of the phase along the undulator is found by taking the derivative of Eq. (21) with respect to $z$ :

$$
\frac{d \psi}{d z}=\left(k+k_{u}\right)-\frac{\omega}{\bar{v}_{z}}
$$

with $\bar{v}_{z}=d z / d \bar{t}$ the average axial velocity of the electron. If one only considers the on-axis case and uses the rms undulator parameter $a_{u}=K / \sqrt{2}$ instead of the peak parameter $K$, this velocity is then:

$$
\bar{v}_{z}=c\left(1-\frac{1+a_{u}^{2}}{2 \gamma^{2}}\right) .
$$

Therefore Eq. (22) is now:

$$
\begin{aligned}
\frac{d \psi}{d z} & \approx\left(k+k_{u}\right)-\frac{\omega}{c}\left(1+\frac{1+a_{u}^{2}}{2 \gamma^{2}}\right) \\
& \approx k_{u}-k \frac{1+a_{u}^{2}}{2 \gamma^{2}} \\
& \approx k_{u}-k_{u} \frac{\gamma_{R}^{2}}{\gamma^{2}}
\end{aligned}
$$

Using a Taylor expansion, Eq. (24a) is derived from Eq. (22), where $\left(1+a_{u}^{2}\right) /\left(2 \gamma^{2}\right)$ is assumed to be small. The on-axis resonance condition described by Eq. (20) with $\theta=0$, is transformed for wavenumber as $k=2 k_{u} \gamma_{R}^{2} /\left(1+a_{u}^{2}\right)$, where $\gamma_{R}$ is the resonant energy. This new convention allows to rewrite Eq. (24b) as Eq. (24c). The relative energy difference being small such that $\Delta \gamma / \gamma_{R} \ll 1$, the second term of Eq. (24c) can be rewritten using the approximation that $\left(\gamma_{r} / \gamma\right)^{2} \approx 1-2 \Delta \gamma / \gamma_{R}$, therefore:

$$
\frac{d \psi}{d z} \approx 2 k_{u}\left(\frac{\Delta \gamma}{\gamma_{R}}\right)
$$

The phase of an electron inside the undulator depends on its energy. Inversely, the energy loss or gain of an electron is related to its phase. The energy exchange rate in the bunch of electrons can be expressed as [10]:

$$
\frac{d \gamma}{d t}=-\frac{e E_{s}^{0} a_{u}}{\gamma m_{0} c} \sin \psi
$$

with $E_{s}^{0}$ the transverse electric field at the entrance of the undulator. There are two different configurations. First, electrons with a phase being between $-\pi$ and 0 gain energy, $d \gamma / d t>0$, they are accelerated and so move to the front of the bunch. Otherwise, if the phase of electrons is between 0 and $\pi$, they lose energy, $d \gamma / d t<0$, so they are delayed in the bunch and shifted to the back of the bunch. Electrons are always kept in phase with the radiation field. Therefore the 
beam is divided into different buckets, each one separated from the others by a distance equal to the resonant wavelength. This process is called microbunching and is illustrated in Fig. 6. The $z-y$ configuration space is plotted, from the left to the right, at the undulator entrance, at half-way saturation, and at full saturation. This modulation of the relativistic beam density exponentially increases the intensity of the emitted light. Waves radiated by the electrons are in phase, hence this coherent emission yields a field intensity proportional to the squared number of electrons [11].

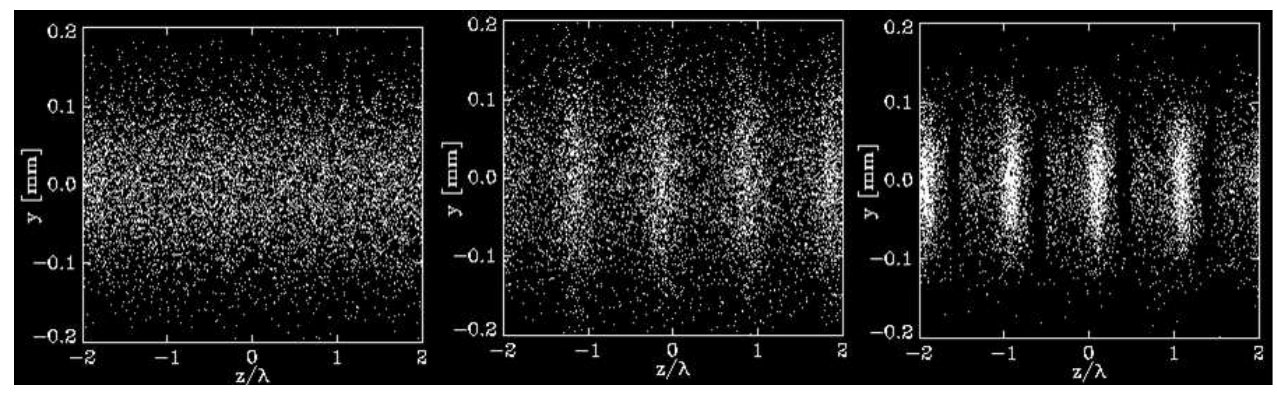

Figure 6: Microbunching of a beam inside of an undulator [8]. 


\subsection{Linac Coherent Light Source}

Around the world, many facilities are planned to be commissioned in the next years, SCSSSPring8 in Japan, the European XFEL project in Germany, and SwissFEL in Switzerland. In the United States of America, the Linear Coherent Light Source (LCLS) was commissioned last year at $\mathrm{SLAC}^{3)}$.

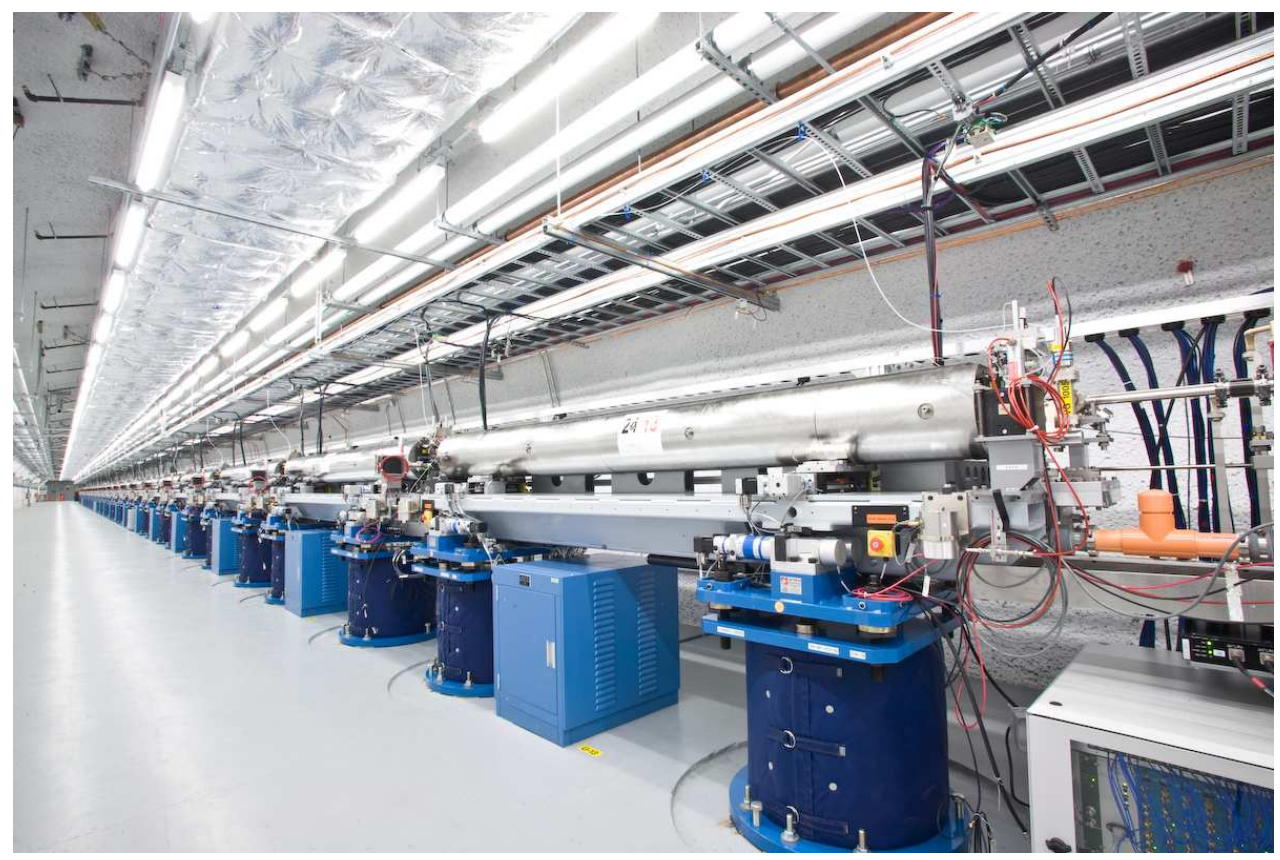

Figure 7: Photograph of the LCLS undulator hall. In the foreground, an undulator covered by a protective shield can be seen on its two pedestals [12].

LCLS uses the last kilometer of the $3 \mathrm{~km}$ SLAC linear accelerator complex to produce an electron beam at an energy of $13.6 \mathrm{GeV}$. This beam is then guided through 33 undulators each $3.4 \mathrm{~m}$ long; the undulator hall is $170 \mathrm{~m}$ long (Fig. 7). At the end of the undulators, light with a wavelength of $15 \mathrm{~nm}$ is emitted in pulses of $100 \mathrm{fs}$ duration. This wavelength can be compared to the size of a DNA helix, or carbon nanotubes. Three X-ray instruments are located in the Near Experimental Hall (NEH), and three others in the Far Experimental Hall (FEH).

For LCLS, X-ray pulses are obtained using the Self-Amplified Spontaneous Emission (SASE) technique. The name of this technique comes from the fact that the initial shot-noise of the beam emits incoherent spontaneous radiation ${ }^{4)}$. The random energy distribution of the electrons generates radiation with jitter around the central wavelength.

3) Located in Menlo Park (California), the SLAC National Accelerator Laboratory is operated by Stanford University for the U.S. Department of Energy (DOE).

4) Coherence of a wave measures its ability to produce interference effects. Coherence can be spatial or temporal. A wave is incoherent when its phase amplitude or phase vary too quickly to produce interference effects. 


\subsection{Seeding techniques for FELs}

A major advantage of SASE is that radiation starts only from noise, no external stimulations are required. However, even if it has an excellent spatial coherence, the temporal coherence is small. The radiation wavelength is much smaller than the beam size, therefore many parts of the bunch of electrons amplify the spontaneous radiation independently from each other. SASE is more adapted for bunches with a short length.

An improvement is to seed the FEL using a laser. This method has been implemented in the High-Gain Harmonic Generation (HGHG) scheme. The beamline is composed of two undulators and one dispersion section, mathematical derivations will be presented in Sec. 2. Inside the first undulator, a laser interacts with the electron beam to modulate its energy at the laser wavelength. The dispersion section then converts this energy modulation to a density modulation. The last undulator enables the beam of electrons to radiate. All the electrons are modulated in energy by the laser; therefore the benefit of this technique is temporally coherence of the generated light. Moreover, the FEL requires shorter undulators to reach saturation [13], so the space needed to build the FEL is reduced, as well as its final cost. Harmonics of $5 \sim 10$ can be generated by the short bunchlets. To achieve higher harmonics, people consider cascade HGHG.

Another mechanism was proposed in 2009 by Gennady Stupakov at SLAC [14]: the Echo Enabled Harmonic Generation (EEHG) concept. Here, like the cascaded HGHG technique, the beam is modulated two times in energy and compressed by a chicane. Firstly, the compression takes place before the second undulator with a large dispersion, and then after the second undulator with a smaller one. This leads to light with higher harmonics than the HGHG technique, called the echo signal. The two modulators ${ }^{5)}$ and the two chicanes (Fig. 8) are followed by a third undulator, also called a radiator, where the harmonically bunched beam of electrons bunched, emits light. This light is useful to demonstrate the efficiency of the EEHG method compared to the HGHG method.

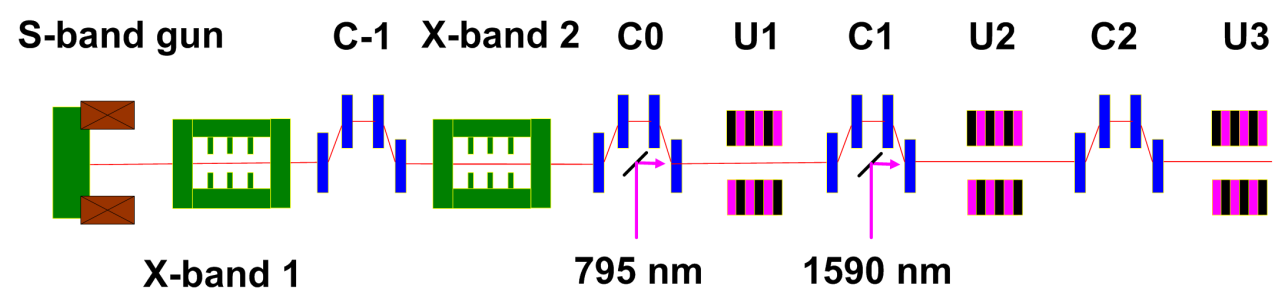

Figure 8: Schema of the EEHG concept [15].

This technique became a focus of interest and several proof-of-principle experiment are currently being executed in different laboratories, for example at Shanghai Synchrotron in China and at SLAC in the USA.

5) An undulator coupled with a laser is called a modulator. 


\section{Theoretical basis of the EEHG concept}

An introduction to the different mathematical concepts of the EEHG technique is presented here. First, the modulation of the beam energy by a laser inside an undulator is explained in details. This is followed by the theoretical working of a chicane in order to understand how it affects the beam phase space. Finally, the different processes occurring during the conversion of an energy modulation into a density modulation are explained.

\subsection{Energy modulation in the first undulator}

At the entrance of the first undulator, the beam profile is assumed to follow a Gaussian distribution, with $E_{0}$ the mean energy and $\sigma_{E}$ its standard deviation. The energy deviation of a single particle of energy $E$, is given by $p=\left(E-E_{0}\right) / \sigma_{E}$. In the longitudinal direction, the initial phase space distribution is given by:

$$
f(p)=\sqrt{2 \pi} N_{0} \exp \left(-\frac{p^{2}}{2}\right),
$$

with $N_{0}$ the number of electrons per unit length of the beam. An example of this initial phase space distribution is shown in Fig. 9. This plot has been produced by a 1D computer simulation code. For the sake of clarity, a uniform distribution of the energy is used. The longitudinal direction is represented by $z$, and $\lambda$ corresponds to the wavelength of the laser used in the first modulator. The two different colors, red and black, are only indicators.

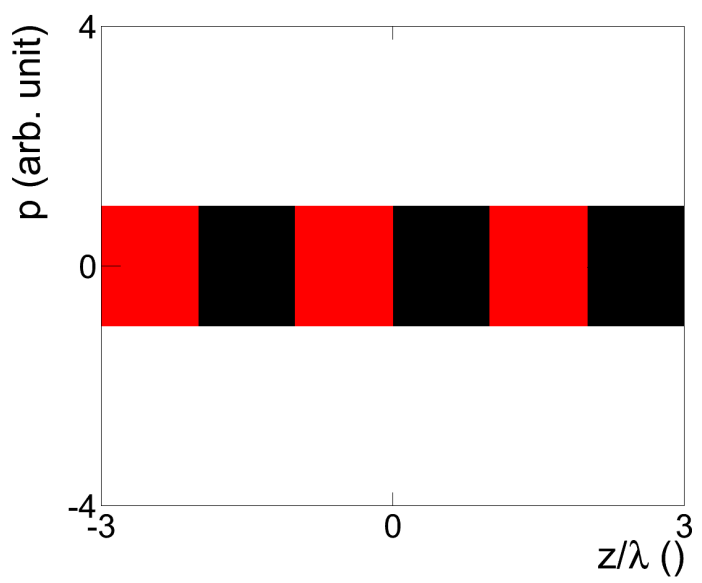

Figure 9: Initial phase space with $\lambda$ the laser wavelength, and $p$ the energy deviation.

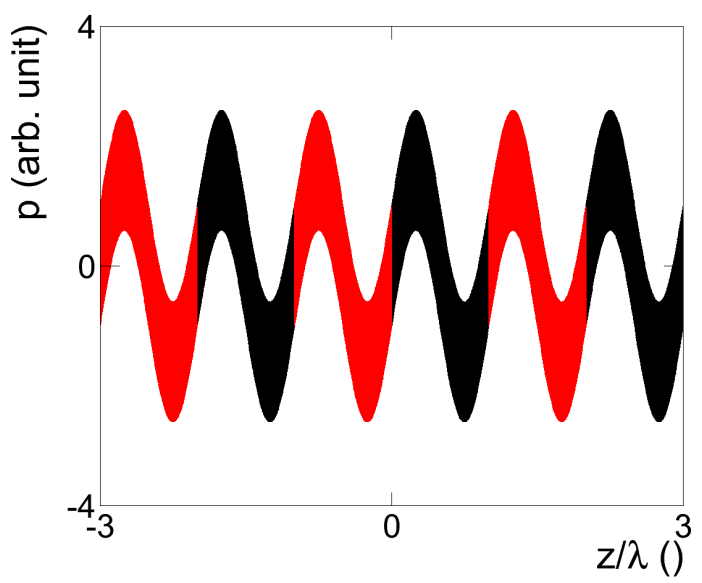

Figure 10: Modulation of the beam energy in the first undulator by the laser.

The next step is the modulation of the beam energy. Laser pulses with the duration on the order of the bunch length are sent into the first undulator. An interaction occurs between the electromagnetic field from the laser and the electrons of the bunch. Similar to the microbunching process, electrons gain or lose energy depending of their relative phase given here by $2 \pi z / \lambda$. The new energy deviation of the electrons is modeled by:

$$
p^{\prime}=p+A_{1} \sin \left(k_{1} z\right)
$$


with $A_{1}=\Delta E_{1} / \sigma_{E}, \Delta E_{1}$ being the amplitude of the modulation, and $k_{1}=\omega_{1} / c$. The other coordinates are kept unchanged. The modulated phase space distribution is shown in Fig. 10. The transfer of energy between the laser field and an electron during this interaction in the undulator is described by [11]:

$$
\frac{d \gamma}{d t}=\frac{e}{m c} E_{x} \beta_{x}
$$

where $E_{x}$ is the laser field polarized in the horizontal plane. The mathematical derivations are presented in Appendix A. Here the laser beam is assumed to have a Gaussian profile. The undulator length $L_{u}$ is considered small compared to the Rayleigh length $Z_{r}$ of the laser, as well as the horizontal and vertical beta functions $\beta_{x}$ and $\beta_{y}$ of the electrons. The amplitude of energy modulation for an electron located at the radial position $r \ll \sigma_{r}$ is [16]:

$$
\Delta \gamma(r)=\sqrt{\frac{P_{L}}{P_{0}}} \frac{K_{u} L_{u}}{\gamma \sigma_{r}}\left[J_{0}\left(\frac{K_{u}^{2}}{4+2 K_{u}^{2}}\right)-J_{1}\left(\frac{K_{u}^{2}}{4+2 K_{u}^{2}}\right)\right] \exp \left(-\frac{r^{2}}{4 \sigma_{r}^{2}}\right)
$$

where $P_{L}$ is the peak laser power, $P_{0}=I_{A} m c^{2} / e$ with $I_{A}$ the Alfvén current, $J_{0}$ and $J_{1}$ are the Bessel functions of the zeroth and first order, and $\sigma_{r}$ is the rms laser spot size in the undulator. Small changes in laser and electron beam sizes during the interaction are neglected. Defining $\zeta=k_{1} z$, the new distribution function after the interaction is:

$$
f(\zeta, p)=\frac{N_{0}}{\sqrt{2 \pi}} \exp \left[-\frac{\left(p-A_{1} \sin \zeta\right)^{2}}{2}\right] .
$$




\subsection{Beam dispersion in a chicane section}

After the undulator, the modulated beam has to be dispersed in order to create energy strips in phase space. This can be realized by a chicane. This element is a bunch compressor, it rearranges the electrons in the longitudinal direction. A chicane is typically composed of four bending magnets, all having the same size and the same strength. The bending angle of the first and fourth magnet is $+\theta$; for the second and third one, the angle is $-\theta$ (Fig. 11).

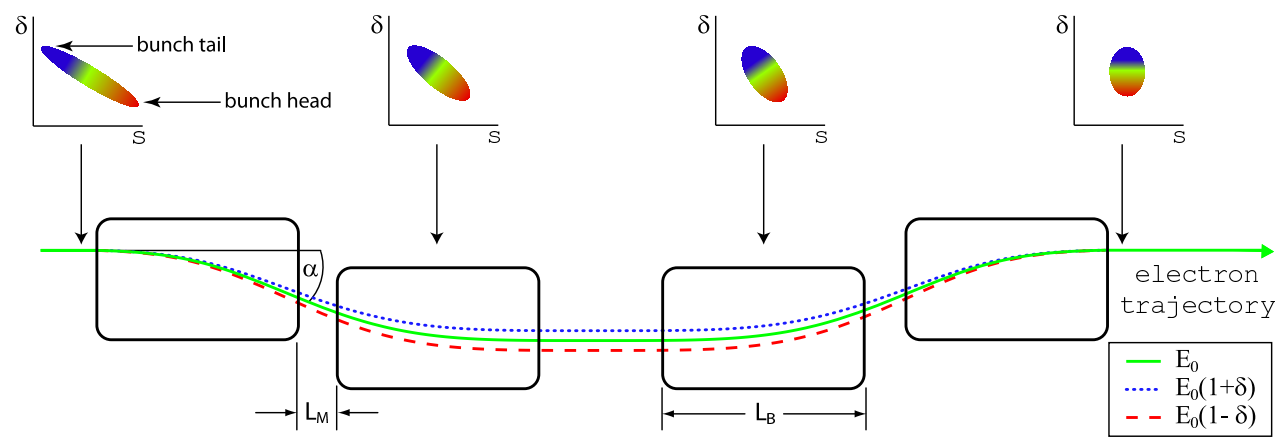

Figure 11: Magnet configuration of a chicane (courtesy of Michael Dunning).

In a chicane, the path length of an electron is dependent on its energy. Larger momentum electrons have a shorter path length, whereas smaller momentum particles have a longer path length. If the bunch is chirped in a linac, the tail of a bunch is composed of high momentum particles, therefore they will move forward. As a result, the bunch is compressed. Using the notation $\delta=p \sigma_{0} / E_{0}$, the longitudinal coordinate is transformed in a chicane as:

$$
\begin{aligned}
z^{\prime}= & z+R_{56} \delta+R_{51} x+R_{52} \frac{d x}{d s}+T_{566} \delta^{2}+T_{511} x^{2}+T_{521} x \frac{d x}{d s} \\
& +T_{522} \frac{d x^{2}}{d s}+T_{533} y^{2}+T_{543} y \frac{d y}{d s}+T_{544} \frac{d y^{2}}{d s}+\ldots
\end{aligned}
$$

The $R_{51}$ and $R_{52}$ terms stem from the field errors and misalignment of the dipoles. In a perfect chicane, only terms up to the second order $T_{566}\left(\approx-3 R_{56} / 2\right)$ term contribute:

$$
z^{\prime}=z+R_{56} \delta+T_{566} \delta^{2}
$$

The energy deviation remains unchanged. In the linear approximation $T_{566} \delta \ll R_{56}$, Eq. (33) can be rewritten in a matrix formalism:

$$
\left(\begin{array}{l}
z^{\prime} \\
\delta^{\prime}
\end{array}\right)=\left(\begin{array}{cc}
1 & R_{56} \\
0 & 1
\end{array}\right)\left(\begin{array}{l}
z \\
\delta
\end{array}\right) .
$$

The momentum compaction factor $R_{56}$ is determined by the following integral [17]:

$$
R_{56}=\int \frac{\eta}{\rho} d s
$$

where $\eta$ is the dispersion and $\rho$ is the instantaneous bending radius. For the symmetric four dipoles chicane Eq. (35) becomes [17]:

$$
R_{56}=2 \theta^{2}\left(L_{M}+\frac{2}{3} L_{B}\right),
$$


with $\theta$ the absolute angle of the bending magnets, $L_{B}$ their length, and $L_{M}$ the distance between two of them.

For the EEHG method, the first chicane has a large $R_{56}^{(1)}$, which creates energy strips in the phase space (Fig. 12).

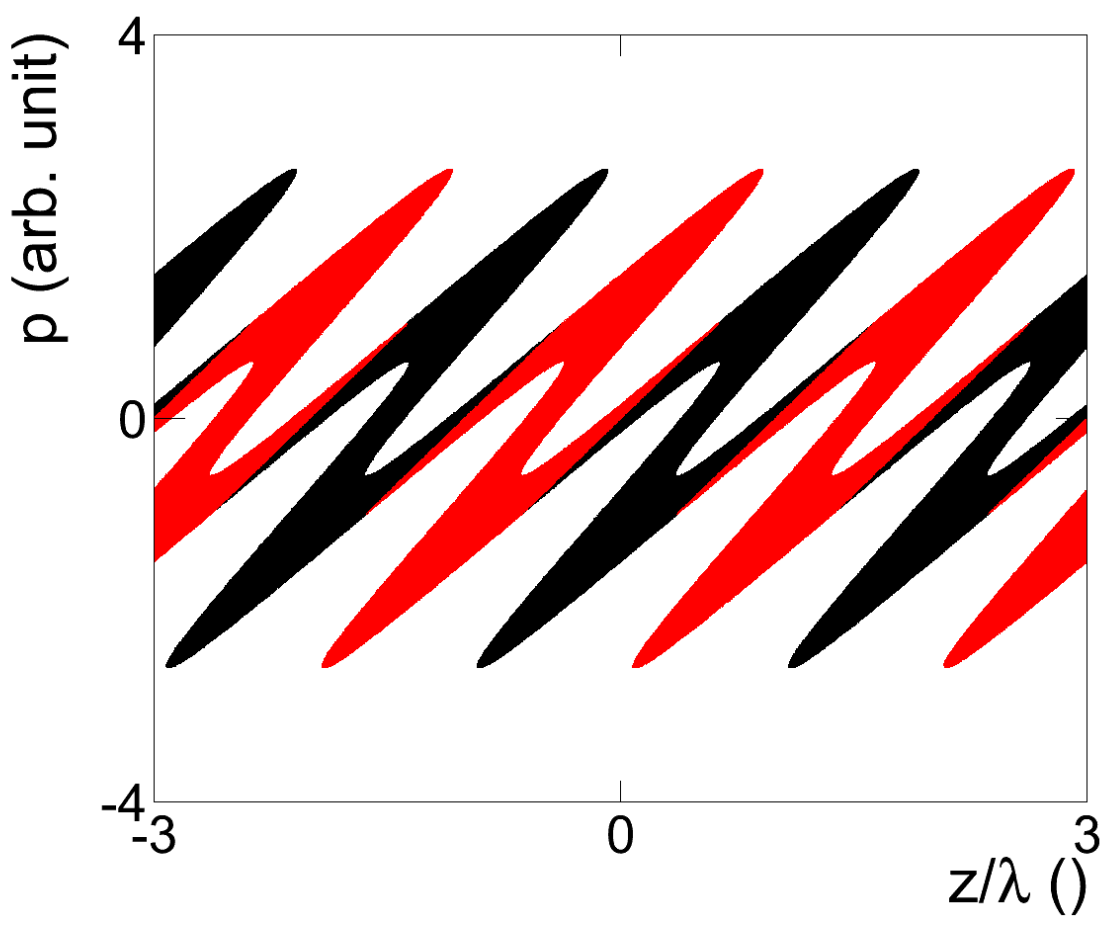

Figure 12: Compression of the bunch by the first chicane.

The new distribution function after this first chicane is:

$$
f(\zeta, p)=\frac{N_{0}}{\sqrt{2 \pi}} \exp \left[-\frac{\left(p-A_{1} \sin \left(\zeta-B_{1} p\right)\right)^{2}}{2}\right],
$$

where $B_{1}=R_{56}^{(1)} k_{1} \sigma_{E} / E_{0}$.

By combining Eqs. (28) and (33), the longitudinal coordinate of an electron, in the first undulator and the first chicane, is given by:

$$
z^{\prime}=z+R_{56}^{(1)} \delta+R_{56}^{(1)} A_{1} \sin \left(k_{1} z\right)+2 T_{566}^{(1)} \delta A_{1} \sin \left(k_{1} z\right)+T_{566}^{(1)} A^{2} \sin ^{2}\left(k_{1} z\right)
$$

in second order of $\delta$. 


\subsection{Density modulation after the first modulation section}

In order to convert the energy bands as seen in Fig. 12 into a density modulation, another undulator and a second chicane are used. This second undulator modulates the energy of the electrons as follows:

$$
p^{\prime}=p+A_{2} \sin \left(k_{2} z+\phi\right),
$$

where $\phi$ is the relative phase of the second laser compared to the first one. The wavelength of the second laser does not need to be the same as the first laser. The resulting phase space distribution is shown in Fig. 13. One notices that for a given position $z$, particles are originating from different initial wavelength ranges, seen here in different colors.

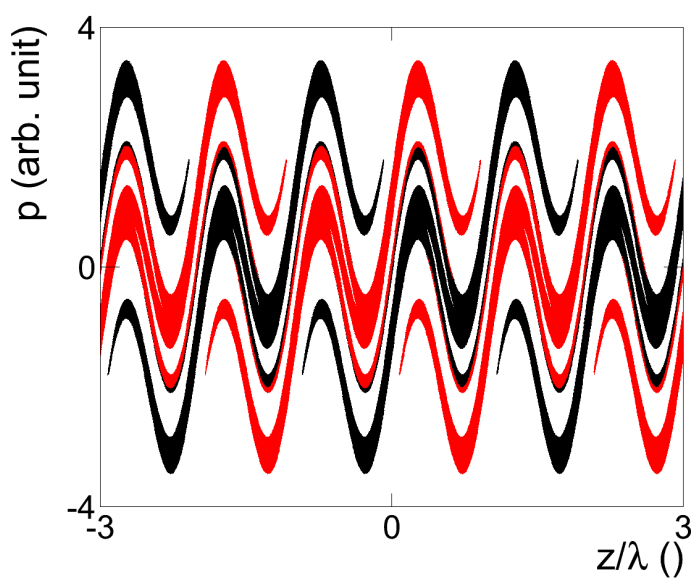

Figure 13: Modulation of the energy by the second undulator.

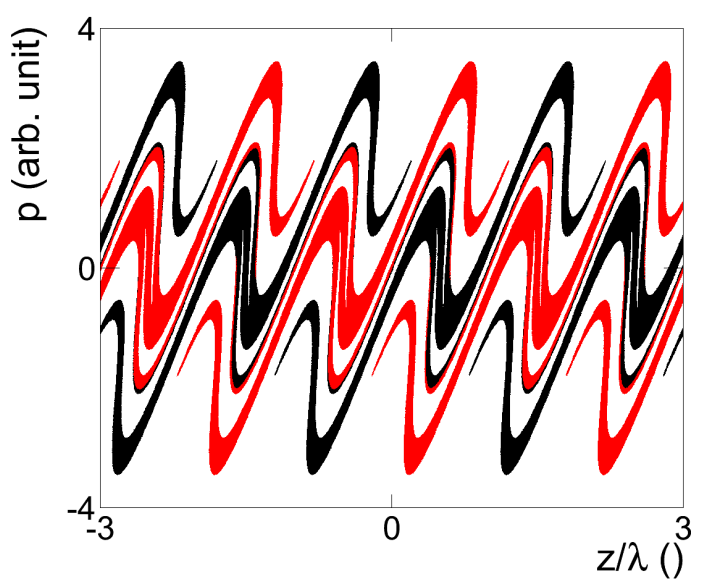

Figure 14: Conversion of the energy modulation into a density modulation by the second chicane.

Eventually, these energy structures are rotated by the second chicane with a weaker dispersive strength of $R_{56}^{(2)}$ compared to the first chicane. The resulting phase space distribution is shown in Fig. 14. The separated energy bands are now upright. The final distribution function is:

$$
\begin{aligned}
f_{f}(\zeta, p)=\frac{N_{0}}{\sqrt{2 \pi}} \exp [ & -\frac{1}{2}\left(p-A_{2} \sin \left(K \zeta-K B_{2} p+\phi\right)-A_{1} \sin \left(\zeta-\left(B_{1}+B_{2}\right) p\right.\right. \\
& \left.\left.\left.+A_{2} B_{1} \sin \left(K \zeta-K B_{2} p+\phi\right)\right)\right)^{2}\right],
\end{aligned}
$$

where $B_{2}=R_{56}^{(2)} k_{1} \sigma_{E} / E_{0}$, and $K$ is the ratio between the two wave vectors $k_{2}$ and $k_{1}$. 
A projection of the phase space on the $z$-axis gives the current distribution after the second chicane, as shown in Fig. 15. The current has been clearly modulated from a flat distribution, it results in a bunched beam in the longitudinal direction. One notices the presence of distinct peaks in one wavelength of the second laser.

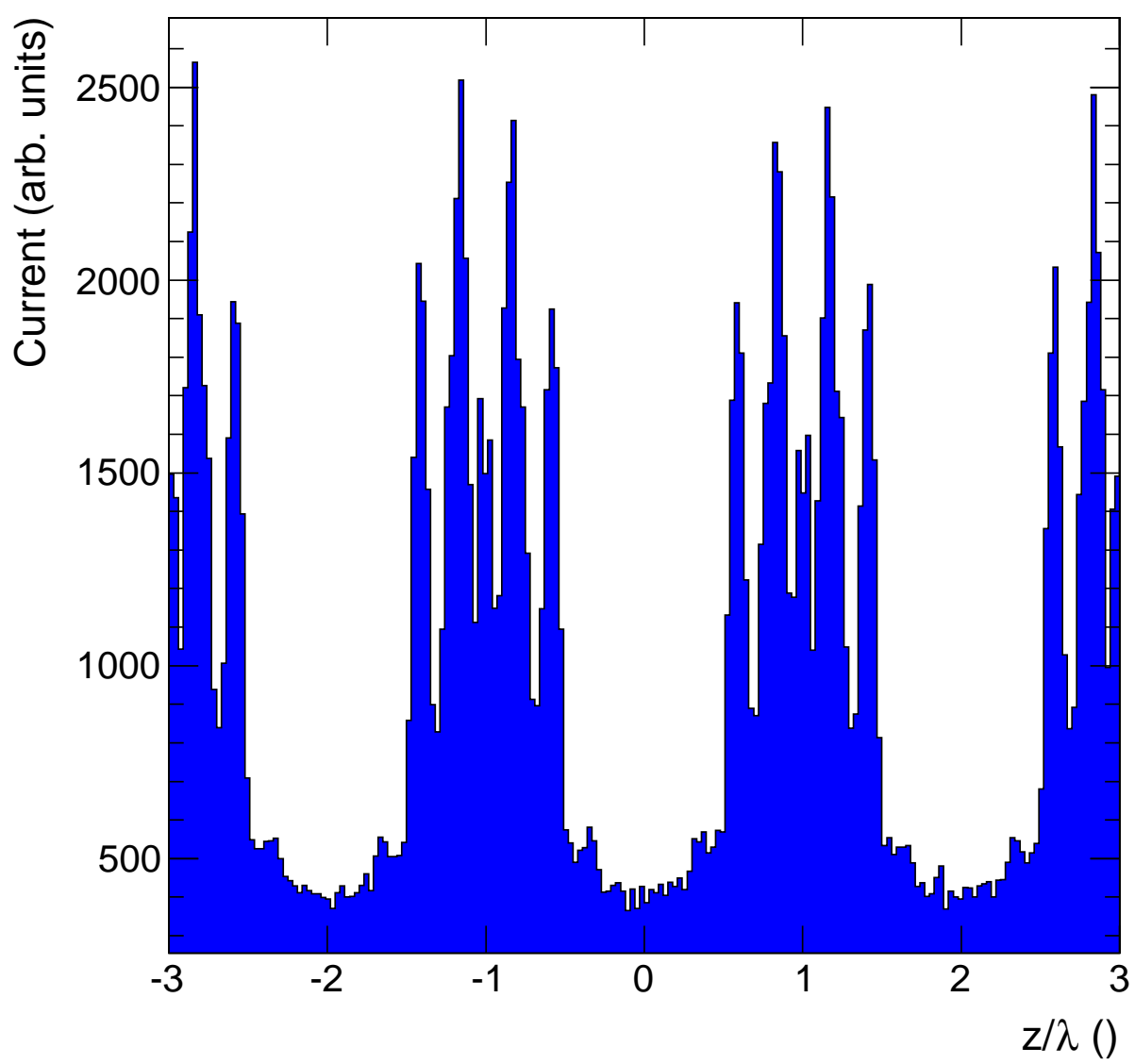

Figure 15: Current distribution after the second chicane.

Finally, a third undulator, called a radiator, is used to generate coherent attosecond X-ray pulses. 


\section{The Echo-7 experiment}

Some design considerations of the Echo-7 proof-of-principle experiment are explained here. Calculations of the configuration parameters for the two undulators, their lasers and the two chicanes are performed. Errors coming from the bend angles of the two chicanes are studied and their angles are optimized in second order. Then the smearing effect due to the emittance of the beam is analyzed by computer simulation. During the installation stage, calculations to determine the spontaneous emission in the two undulators have been performed and are summarized here. Studies on the effect of the second laser phase relative to the first are detailed. Eventually, the jitter in the RF structures is measured.

\subsection{Choice of the parameters}

The Next Linear Collider Test Accelerator ${ }^{6)}$ (NLCTA) at SLAC has been modified to build a proof-of-principle experiment for the EEHG effect: the Echo-7 experiment. It is the most suitable place at SLAC to build such an experiment. The Echo-7 experiment has been designed and optimized to generate radiation tuned at the seventh harmonic of the second laser. Lower and higher harmonics are still achievable. Parameters have been calculated for operation at the fourth, fifth, and fifteenth harmonics as well. A photograph of the beamline is shown in Fig. 16, where two chicanes are visible (undulators were not installed at that time). Modifications in the current beamline of the NLCTA are minor, only three chicanes ${ }^{7)}$ and three undulators had to be installed. It will produce an energy modulation with a spacing of $224 \mathrm{~nm}$, from an electrons beam of $120 \mathrm{MeV}$.

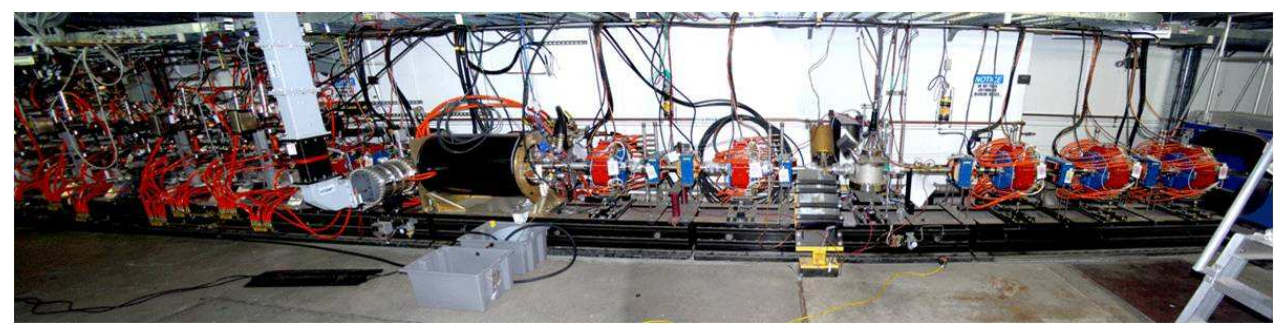

Figure 16: Photograph of the Echo-7 experiment during its installation at the NLCTA.

Parameters for the two undulators and their laser $\left(A_{1}, A_{2}\right)$, and for the two chicanes $\left(B_{1}, B_{2}\right)$ have to be chosen in order to generate the seventh harmonic. A Fourier transform of the current distribution (Fig. 15) can give the spectrum of the light radiated by the final undulator. However an easier and more accurate way to look at the amplitude of the $k^{\text {th }}$ harmonic, is to compute directly the bunching factor $b_{k}$. This is the ratio between the average and the peak density of the beam.

6) Physicists at the NLCTA are in charge of the accelerator R\&D to develop new technologies for the International Linear Collider (ILC).

7) The zeroth chicane is necessary to bend the beam in order to send laser pulses inside of the first undulator. 
For the $k^{\text {th }}$ harmonic, it is defined as follows:

$$
b_{k}=\frac{1}{N_{0}}\left|\left\langle e^{-i k \zeta} N(\zeta)\right\rangle\right|
$$

where $N_{0}$ is the number of electrons in the bunch. The beam density $N(\zeta)$ is computed by integrating Eq. (40):

$$
N(\zeta)=\int_{-\infty}^{+\infty} f_{f}(\zeta, p) d p
$$

Averaging over the coordinate $\zeta$ yields:

$$
\langle f\rangle=\lim _{L \rightarrow+\infty} \frac{1}{2 L} \int_{-L}^{+L} f(\zeta) d \zeta .
$$

The bunching factor is nonzero if the following condition is fulfilled [18]:

$$
k=n+K m,
$$

with $n, m \in \mathbb{Z}$, and $K=k_{2} / k_{1}$. Multiplying Eq. (44) by $k_{1}$ gives the echo wavenumber $k_{E}$ of the final modulation:

$$
k_{E}=n k_{1}+m k_{2}
$$

Using the condition of Eq. (44), the bunching factor transforms to:

$$
\begin{aligned}
b_{k}= & \mid \exp \left(-\frac{\left(n B_{1}+(K m+n) B_{2}\right)^{2}}{2}\right) \times J_{m}\left[-(K m+n) A_{2} B_{2}\right] \\
& \times J_{n}\left[-A_{1}\left(n B_{1}+(K m+n) B_{2}\right)\right] \mid
\end{aligned}
$$

where $J_{m, n}$ are Bessel functions of the first kind defined by:

$$
J_{n}(z)=\frac{1}{2 \pi i} \oint \exp \left(\frac{z(t-1)}{2 t} t^{-n-1}\right) d t .
$$

A necessary condition to maximize the bunching factor is $n= \pm 1$. For our following studies, $n$ is arbitrarily fixed to -1 . Eq. (46) now reads:

$$
\begin{aligned}
b_{k}= & \mid \exp \left(-\frac{\left(-B_{1}+(K m-1) B_{2}\right)^{2}}{2}\right) \times J_{m}\left[-(K m-1) A_{2} B_{2}\right] \\
& \times J_{1}\left[-A_{1}\left(-B_{1}+(K m-1) B_{2}\right)\right] \mid
\end{aligned}
$$

where the following property of the Bessel functions were used:

$$
J_{n}(z)=(-1)^{n} J_{n}(z) .
$$


One has to choose the four parameters $A_{1}, A_{2}, B_{1}$ and $B_{2}$ to maximize Eq. (48). Both $A_{1}$ and $A_{2}$ are related to the energy modulation in the two undulators. At the NLCTA, the wavelength of the first laser is fixed at $\lambda=795 \mathrm{~nm}$. In order to avoid having two resonances for the bunching factor too close to each other for different $m$, the ratio between the wavelengths of the two lasers is chosen to be $K=1 / 2$. Other properties of the lasers are listed in Tab. 1.

\begin{tabular}{llll}
\hline \hline & $\lambda$ & $P$ & $w_{0}$ \\
\hline Laser 1 & $795 \mathrm{~nm}$ & $7.4 \mathrm{MW}$ & $1.8 \mathrm{~mm}$ \\
Laser 2 & $1590 \mathrm{~nm}$ & $3.8 \mathrm{MW}$ & $1.8 \mathrm{~mm}$ \\
\hline \hline
\end{tabular}

Table 1: Parameters of the two lasers for Echo-7.

Here $\lambda$ is the wavelength of the laser, $P$ represents the peak power, $w_{0}$ its spot size at the waist. For the two undulators, the material of choice is Nd-Fe-B: their parameters are listed in Tab. 2.

\begin{tabular}{llllll}
\hline \hline & $N$ & $\lambda_{u}$ & $K$ & $B_{0}$ & Gap \\
\hline Undulator 1 & 10 & $3.3 \mathrm{~cm}$ & 1.80 & $0.58 \mathrm{~T}$ & $12.4 \mathrm{~mm}$ \\
Undulator 2 & 10 & $5.5 \mathrm{~cm}$ & 2.07 & $0.40 \mathrm{~T}$ & $25.0 \mathrm{~mm}$ \\
\hline \hline
\end{tabular}

Table 2: Parameters of the two undulators for Echo-7.

The symbol $N$ is the number of periods, $\lambda_{u}$ is the period, $K$ is the deflection parameter defined by Eq. (9), and $B_{0}$ is the undulator peak field. With these parameters, energy modulations can be computed. In the first undulator $\Delta E_{1}=30 \mathrm{keV}$, and in the second $\Delta E_{2}=40 \mathrm{keV}$. The NLCTA electron beam has a charge between $20 \mathrm{pC}$ and $40 \mathrm{pC}$, an energy $E_{0}$ of $120 \mathrm{MeV}$, and a slice energy spread $\sigma_{E}$ of $10 \mathrm{keV}$. Therefore, we obtain $A_{1}=3$ and $A_{2}=4$.

To determine the configuration of the two chicanes $\left(B_{1}, B_{2}\right)$, Eq. (44) has to be solved, with $K=1 / 2$. One finds $m=9$ for the seventh harmonic, i.e. $k_{E}=3.5$ with respect to the first laser. For a large $m$, the first part of Eq. (48) is maximized when its argument is equal to $m+0.81 m^{1 / 3}$. The value of the Bessel function $J_{m}$ is now $0.67 / m^{1 / 3}$ [19]. The necessary condition for the parameter $B_{2}$ is the following:

$$
(K m-1) A_{2} B_{2}=m+0.81 m^{1 / 3} .
$$

For Echo-7, one obtains $B_{2}=0.7632$. The next step is to find the corresponding value for the parameter $B_{1}$. To maximize the second part of Eq. (48), a new variable $\xi=B_{1}-(K m-1) B_{2}$ is introduced. The product $\exp \left(\xi^{2} / 2\right) \times J_{1}\left[A_{1} \xi\right]$ is differentiated with respect to $\xi$, and set to zero to find the value of $\xi$ which maximizes it. The condition on $B_{1}$ is:

$$
A_{1}\left(J_{0}\left[A_{1} \xi\right]-J_{2}\left[A_{1} \xi\right]\right)-2 \xi J_{1}\left[A_{1} \xi\right]=0
$$


This equation has an infinite number of solutions. Four of them are plotted in Fig. 17 in the range $\xi \in[-2,+2]$. Solutions can be numerically found thanks to a script written in Python. For our studies, three roots have been selected. Final solutions are listed in Tab. 3.

\begin{tabular}{lllll}
\hline & $A_{1}$ & $A_{2}$ & $B_{1}$ & $B_{2}$ \\
\hline Solution 1 & 3 & 4 & 0.76 & 1.06 \\
Solution 2 & 3 & 4 & 0.76 & 2.14 \\
Solution 3 & 3 & 4 & 0.76 & 3.20 \\
\hline \hline
\end{tabular}

Table 3: Parameters for the lasers and the chicanes.

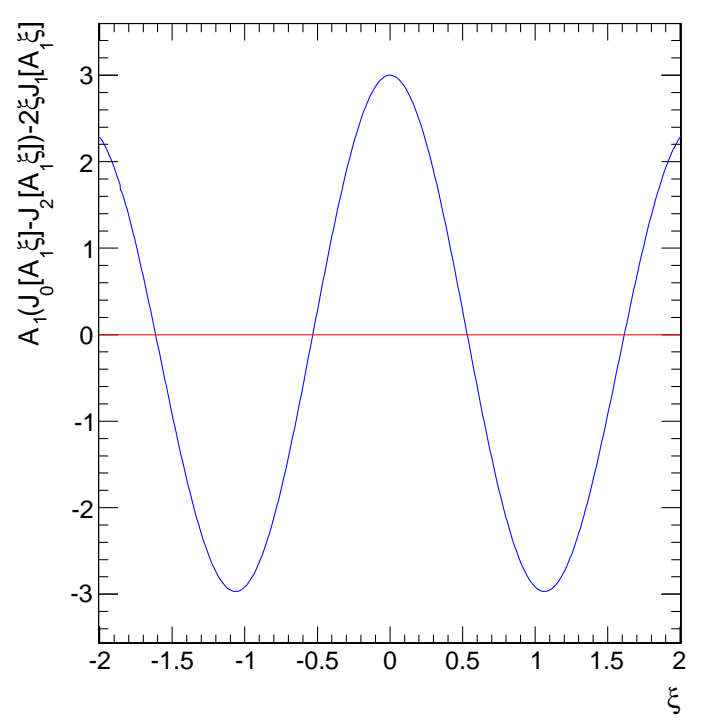

Figure 17: Four solutions of Eq. (51) in the range $\xi \in[-2,+2]$.

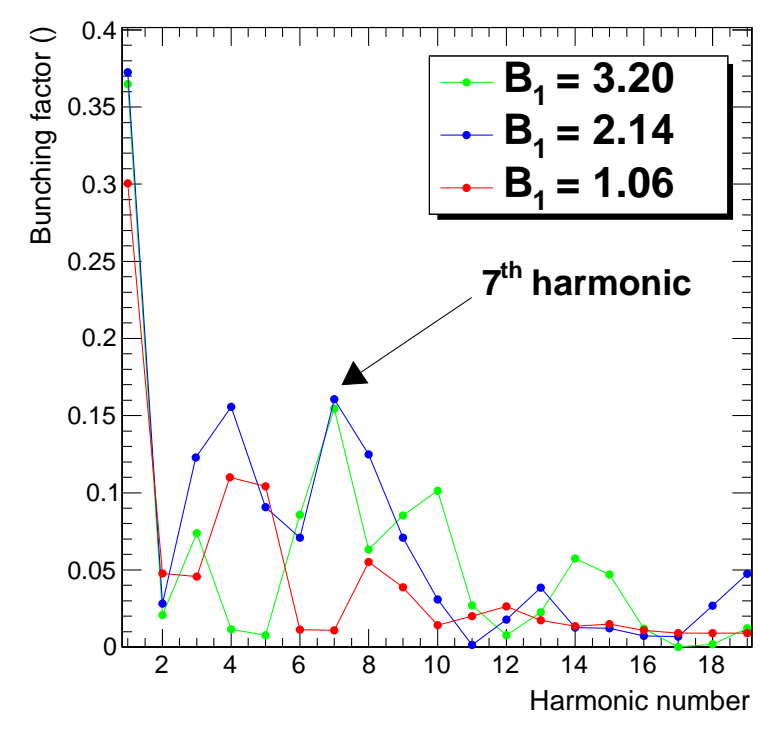

Figure 18: Comparison of the bunching factor for different solutions $B_{1}$ optimized for the seventh harmonic.

A 1D simulation code has been developed in Python to study these three different solutions. A bunch of $2 \times 10^{5}$ macro-particles is generated with a Gaussian distribution. Electrons inside of the two modulators and the two chicanes are tracked using electron coordinates $(z, p)$. In the longitudinal direction, electrons are kept in the range of $\left[-3 \lambda_{1},+3 \lambda_{1}\right]$ to increase the speed of simulations, with $\lambda_{1}$ the wavelength of the first laser. The EEHG method uses two lasers to modulate the beam energy. These pulses overlap the beam on a distance more than $6 \lambda$. At the end of the entire process, particles from the rear and from the front of bunch will be present in the initial range of $\left[-3 \lambda_{1},+3 \lambda_{1}\right]$. Therefore periodic boundary conditions have to be applied here. 
Bunching factors for the three solutions for the $B_{1}$ parameter are shown in Fig. 18, for harmonic numbers between 1 and 19. These results are also compared in Tab. 4 to the analytical solutions derived from Eq. (48). The solution for $B_{1}=1.06$ does not give a resonance at the seventh harmonic, $b_{7}=1.1 \%$, therefore this one can be eliminated from the set of possible configurations for the first chicane. For the two other solutions, $B_{1}=2.14$ and $B_{1}=3.20$, there is a peak at the seventh harmonic and $b_{7} \approx 16 \%$. These results are confirmed by theory. One can notice that peaks at other harmonics are also present for the bunching factor. For the following studies, we keep $B_{1}=2.14$ and $B_{1}=3.20$ solutions for the first chicane. For the second chicane, $B_{2}$ is fixed at 0.7632 .

\begin{tabular}{lll}
\hline \hline & 1D simulation & Theory \\
\hline$B_{1}=3.20$ & $15.3 \%$ & $15.4 \%$ \\
$B_{1}=2.14$ & $16.0 \%$ & $15.4 \%$ \\
$B_{1}=1.06$ & $1.1 \%$ & $2.6 \%$ \\
\hline \hline
\end{tabular}

Table 4: Comparison of $b_{7}$ between 1D simulation and theory for different $B_{1}$. 


\subsection{Optimization of the parameters for the two chicanes}

The configuration parameters for the two chicanes have to be optimized with computer simulations closer to reality. The 6D tracking code elegant ${ }^{8)}$ (ELEctron Generation ANd Tracking) has been chosen for this task. This code generates and tracks particles in the different elements composing the beamline of an accelerator.

For our studies, the entire beamline of the Echo-7 experiment has been modeled. More details about the magnets composing this beamline are listed in Appendix B. Parameters for the simulations are listed in Tab. 5. For the two undulators, the non-adaptive Runge-Kutta method over 100 steps has been chosen to improve accuracy of the integration.

\begin{tabular}{lll}
\hline \hline Number of macro-particles & & $2 \times 10^{5}$ \\
Initial distribution & & Gaussian \\
Total bunch charge & & $25 \mathrm{pC}$ \\
Central momentum & $\beta \gamma$ & $234.8 \mathrm{MeV} / \mathrm{c}$ \\
Fractional momentum spread & $\delta$ & $83.3 \mathrm{eV} / \mathrm{c}$ \\
Horizontal emittance & $\epsilon_{x}$ & $8 \mu \mathrm{m} \cdot \mathrm{mrad}$ \\
Vertical emittance & $\epsilon_{y}$ & $8 \mu \mathrm{m} \cdot \mathrm{mrad}$ \\
Bunch length & $\sigma_{s}$ & $4 \mu \mathrm{m}$ \\
Initial horizontal beta function & $\beta_{x}$ & 6.8 \\
Initial vertical beta function & $\beta_{y}$ & 2.0 \\
\hline \hline
\end{tabular}

Table 5: Parameters used for computer simulations.

The two chicanes used for this experiment are composed of four bending magnets with a length $L_{B}=11.2 \mathrm{~cm}$, and a distance between them of $L_{M}=30 \mathrm{~cm}$. In elegant, the order of the matrices and the edge matrices is fixed at 2. For the solutions found in Sec. 3.1, $R_{56}$ and the corresponding bending angles $\theta$ of these two chicanes are listed in Tab. 6 .

\begin{tabular}{lllll}
\hline \hline & $R_{56}^{(1)}$ & $\theta_{1}$ & $R_{56}^{(2)}$ & $\theta_{2}$ \\
\hline$B_{1}=3.20$ & $4.80 \mathrm{~mm}$ & $80.1 \mathrm{mrad}$ & $1.14 \mathrm{~mm}$ & $39.1 \mathrm{mrad}$ \\
$B_{1}=2.14$ & $3.20 \mathrm{~mm}$ & $65.4 \mathrm{mrad}$ & $1.14 \mathrm{~mm}$ & $39.1 \mathrm{mrad}$ \\
\hline \hline
\end{tabular}

Table 6: Momentum compaction factor and angles for the two chicanes.

For the chicanes, one source of possible errors is the magnetic field variation of the different magnets due to their misalignment. This can modify the $B_{1}$ parameters and therefore reduce the bunching factor at the end of the experiment. The magnetic field inside of a chicane is related to the bend angle $\theta$ of the four bending magnets. This is changed for the four magnets at the same time by a fixed percentage between $-5 \%$ and $+5 \%$. The results for the bunching factor of the seventh harmonic, $b_{7}$, are shown in Fig. 19 for the two chicanes.

8) Binaries and documentation are available here:

http://www.aps.anl.gov/Accelerator_Systems_Division/Operations_Analysis/software.shtml\#elegant. 
The two red curves represent the dependence of the bunching factor $b_{7}$ on magnet angle for the first chicane. For both solutions $B_{1}=2.13$ and $B_{1}=3.20$, the bunching factor is maximized when the bend angle $\theta$ is shifted by $+1 \%$ from its initial value found by solving Eqs. (50) and (51). For the second chicane, plotted in blue, the optimized value for the angle $\theta$ is shifted by $-1 \%$ from the analytical solution.

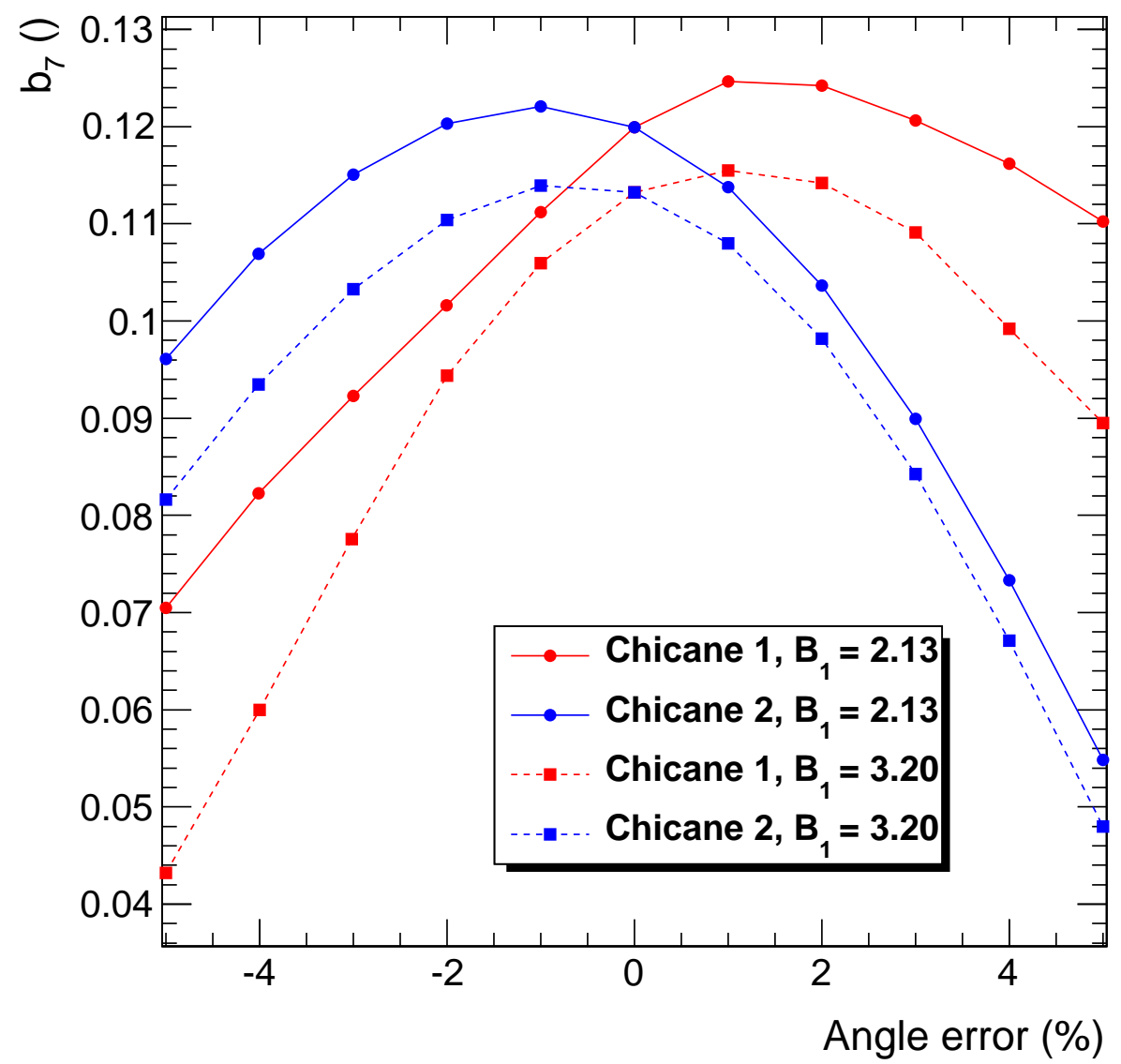

Figure 19: Dependence of the bunching factor on the magnet bend angle for the two chicanes.

The optimized angles at the first order, and their corresponding $R_{56}$ are listed in Tab. 7 .

\begin{tabular}{lllll}
\hline & $R_{56}^{(1)}$ & $\theta_{1}$ & $R_{56}^{(2)}$ & $\theta_{2}$ \\
\hline \hline$B_{1}=3.20$ & $4.90 \mathrm{~mm}$ & $80.9 \mathrm{mrad}$ & $1.12 \mathrm{~mm}$ & $38.7 \mathrm{mrad}$ \\
$B_{1}=2.14$ & $3.26 \mathrm{~mm}$ & $66.1 \mathrm{mrad}$ & $1.12 \mathrm{~mm}$ & $38.7 \mathrm{mrad}$ \\
\hline
\end{tabular}

Table 7: Optimized parameters in first order for the two chicanes.

The bunching factor is also weaker with elegant simulations, around $12 \%$, than the $16 \%$ estimated with the 1D code. This stems from the fact that in elegant, the laser is modeled with a finite beam size. Particles on the outside of the bunch are receiving less energy than those close to the center. This effect, as well as the emittance effect, are not modeled here. 
The stability of the solutions presented in Tab. 7 are studied. Newton's method was used for this task. This method is also useful to optimize these solutions in second order with few iterations. This algorithm enables one to find the maximum of the bunching factor as a function of the bend angles of the two chicanes $\theta_{1}$ and $\theta_{2}$.

The starting point is the vector $\vec{\theta}_{0}=\left(\theta_{1}, \theta_{2}\right)$. The gradient $\nabla b_{7}\left(\theta_{1}, \theta_{2}\right)$ is calculated as follows:

$$
\nabla b_{7}\left(\theta_{1}, \theta_{2}\right)=\left(\frac{\partial}{\partial \theta_{1}} b_{7}\left(\theta_{1}, \theta_{2}\right), \frac{\partial}{\partial \theta_{2}} b_{7}\left(\theta_{1}, \theta_{2}\right)\right) .
$$

Then the Hessian matrix $H f\left(\theta_{1}, \theta_{2}\right)$ defined by

$$
H f\left(\theta_{1}, \theta_{2}\right)=\left(\begin{array}{cc}
\frac{\partial^{2}}{\partial \theta_{1}^{2}} b_{7}\left(\theta_{1}, \theta_{2}\right) & \frac{\partial^{2}}{\partial \theta_{1} \partial \theta_{2}} b_{7}\left(\theta_{1}, \theta_{2}\right) \\
\frac{\partial^{2}}{\partial \theta_{2} \partial \theta_{1}} b_{7}\left(\theta_{1}, \theta_{2}\right) & \frac{\partial^{2}}{\partial \theta_{2}^{2}} b_{7}\left(\theta_{1}, \theta_{2}\right)
\end{array}\right),
$$

is computed. The bunching factor function $b_{7}\left(\theta_{1}, \theta_{2}\right)$ being continuous on its domain of definition, gives the following condition:

$$
\frac{\partial^{2}}{\partial \theta_{1} \partial \theta_{2}} b_{7}\left(\theta_{1}, \theta_{2}\right)=\frac{\partial^{2}}{\partial \theta_{2} \partial \theta_{1}} b_{7}\left(\theta_{1}, \theta_{2}\right) .
$$

Therefore, the Hessian matrix is symmetric. The point $\vec{\theta}_{k+1}$ is computed recursively from the previous one $\vec{\theta}_{k}$, starting at $\vec{\theta}_{0}$, by using the following relation:

$$
\vec{\theta}_{k+1}=\vec{\theta}_{k}-\nabla b_{7}\left(\vec{\theta}_{k}\right)\left[H f\left(\vec{\theta}_{k}\right)\right]^{-1},
$$

where $[\ldots]^{-1}$ represents the inverse of the matrix, here for a $2 \times 2$ matrix:

$$
\left(\begin{array}{ll}
a & b \\
c & d
\end{array}\right)^{-1}=\frac{1}{a d-b c}\left(\begin{array}{cc}
d & -b \\
-c & a
\end{array}\right) \text {. }
$$

The new angles are then computed. This iterative scheme is stopped when the gradient is smaller than a threshold value. For more stability, change of the angles for each step is upper limited to avoid large gaps. Derivatives are approximated by the following finite differences:

$$
\begin{aligned}
\frac{\partial}{\partial \theta_{1}} b_{7}\left(\theta_{1}, \theta_{2}\right) \approx & \frac{b_{7}\left(\theta_{1}+h, \theta_{2}\right)-b_{7}\left(\theta_{1}, \theta_{2}\right)}{2 h}, \\
\frac{\partial}{\partial \theta_{2}} b_{7}\left(\theta_{1}, \theta_{2}\right) \approx & \frac{b_{7}\left(\theta_{1}, \theta_{2}+h\right)-b_{7}\left(\theta_{1}, \theta_{2}\right)}{2 h}, \\
\frac{\partial^{2}}{\partial \theta_{1}^{2}} b_{7}\left(\theta_{1}, \theta_{2}\right) \approx & \frac{b_{7}\left(\theta_{1}+h, \theta_{2}\right)-2 b_{7}\left(\theta_{1}, \theta_{2}\right)+b_{7}\left(\theta_{1}-h, \theta_{2}\right)}{2 h}, \\
\frac{\partial^{2}}{\partial \theta_{2}^{2}} b_{7}\left(\theta_{1}, \theta_{2}\right) \approx & \frac{b_{7}\left(\theta_{1}, \theta_{2}+h\right)-2 b_{7}\left(\theta_{1}, \theta_{2}\right)+b_{7}\left(\theta_{1}, \theta_{2}-h\right)}{2 h}, \\
\frac{\partial^{2}}{\partial \theta_{1} \partial \theta_{2}} b_{7}\left(\theta_{1}, \theta_{2}\right) \approx & \frac{b_{7}\left(\theta_{1}+h, \theta_{2}+h\right)-b_{7}\left(\theta_{1}+h, \theta_{2}-h\right)}{4 h^{2}} \\
& +\frac{-b_{7}\left(\theta_{1}-h, \theta_{2}+h\right)-b_{7}\left(\theta_{1}-h, \theta_{2}-h\right)}{4 h^{2}}, \\
\frac{\partial^{2}}{\partial \theta_{2} \partial \theta_{1}} b_{7}\left(\theta_{1}, \theta_{2}\right) \approx & \frac{b_{7}\left(\theta_{1}+h, \theta_{2}+h\right)-b_{7}\left(\theta_{1}+h, \theta_{2}-h\right)}{4 h^{2}} \\
& +\frac{-b_{7}\left(\theta_{1}-h, \theta_{2}+h\right)-b_{7}\left(\theta_{1}-h, \theta_{2}-h\right)}{4 h^{2}} .
\end{aligned}
$$


A script has been written in Python to implement the Newton's algorithm. It automatically runs elegant simulations, and analyzes the results. The factor $h$ is equal to $0.5 \mathrm{mrad}$. The stopping condition is $\|\nabla f\|<0.5$, and the upper limit for the angle change is 3 mrad. To study the convergence of this method, three different starting points have been chosen. The first one is $\vec{\theta}=\left(\theta_{1}, \theta_{2}\right)$ coming from Eqs. (50) and (51); the second one is $\vec{\theta}=\left(\theta_{1}^{\max }, \theta_{2}\right)$, where $\theta_{1}^{\max }$ is the maximized angle in first order for the first chicane coming from Tab. 7; eventually the last point is $\vec{\theta}=\left(\theta_{1}, \theta_{2}^{\max }\right)$, with $\theta_{2}^{\max }$ is the maximized angle in first order for the second chicane.

For $B_{1}=3.20$, evolution of the iterative vector $\vec{\theta}_{k}$ is shown in Fig. 20 from the three different starting points. The algorithm converges quickly, usually with six to eight iterations. At the end of this process, the optimized angles in second order are $\theta_{1}=80.9 \mathrm{mrad}$ and $\theta_{2}=39.1 \mathrm{mrad}$. For these, the bunching factor for the seventh harmonic is $b_{7}=12 \%$.

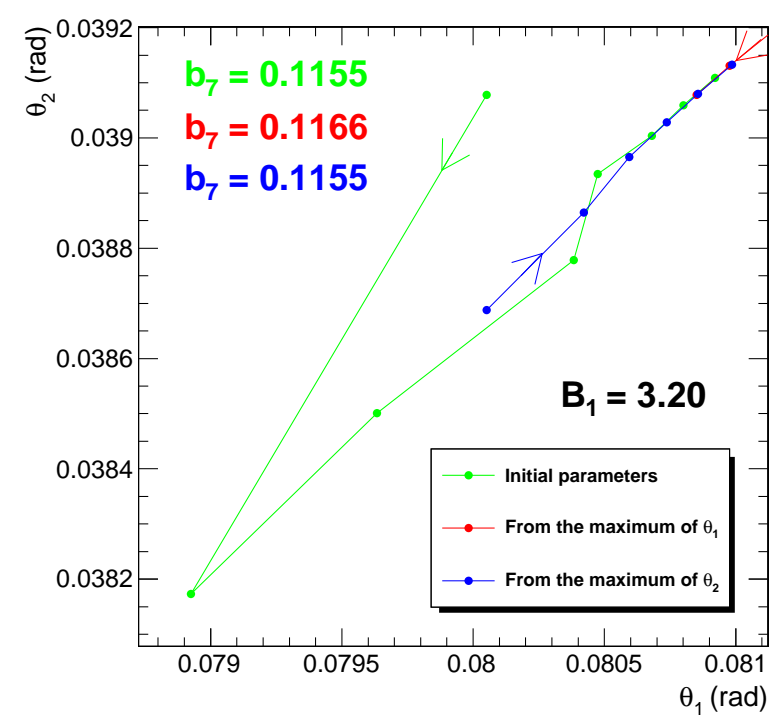

Figure 20: Newton's algorithm for $B_{1}=$ 3.20 , starting from three different points.

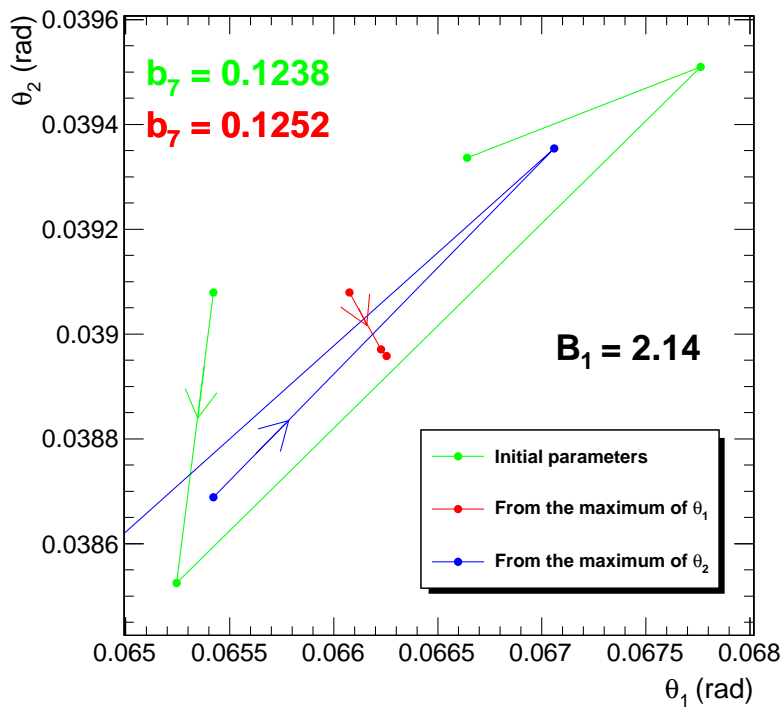

Figure 21: Newton's algorithm for $B_{1}=$ 2.14, starting from three different points.

The same calculations were performed for $B_{1}=2.14$, see Fig. 21. The bunching factor $b_{7}$ is also equal to $12 \%$ for $\theta_{1}=66 \mathrm{mrad}$ and $\theta_{2}=39 \mathrm{mrad}$. However this time, the algorithm does not converge to a maximum when starting from the maximum of $\theta_{2}$; even if the angle shift is limited, Newton's method converges to a minimum of the bunching factor. 


\subsection{Smearing effect due to the emittance}

One of the most significant advantages of the EEHG method compared to HGHG is the possibility to produce a light with higher harmonics. This is the result of the density modulation of the beam created by the two undulators and the two chicanes. The most important step of this process happens in the first chicane. There, the beam is over-compressed due to the large $R_{56}$, and energy strips are produced. After the second chicane, one can notice that these energy bands are now upright in phase space (Fig. 14). The harmonic produced is directly related to the spacing between two consecutive bands, and so determines the properties of the light at the end of the radiator. In the projection on the $z$-axis (Fig. 15), the spacing between two different peaks corresponds to the space between two different energy bands in phase space.

The challenge of the EEHG technique is to preserve this fine structure up to the radiator. A decrease of the spacing between two bands, or even worst the overlapping of two of them, will completely reduce the efficiency of the EEHG method to obtain high harmonics. There are many other processes which can destroy this structure, and so to wash out the energy bands. Some of them have already been studied, for example the misalignment of the dipoles or the geometric aberration.

The role of the emittance has to be studied in order to analyze its effect on the bunching factor. Computer simulations have been performed using the elegant code. The beamline has been set up for two different configurations of the chicanes. The first one is the solution with $B_{1}=2.14$, the second one with $B_{1}=3.20$. These two solutions are optimized for the seventh harmonic of the second laser. Other parameters are the same as in previous simulations. The emittance varies between $0.1 \mathrm{~mm} \cdot \mathrm{mrad}$ and $15 \mathrm{~mm} \cdot \mathrm{mrad}$. Both horizontal and vertical emittances are changed at the same time. This range corresponds to the real values we can expect from the beamline at the NLCTA, taking into account the different elements between the gun and the beginning of the Echo-7 experiment. Results have been processed by the SDDS toolkit to extract the $z$ coordinate of each electron. Then they have been analyzed with a Python script to compute directly the bunching factor for the seventh harmonic. The results are presented in Fig. 22. 


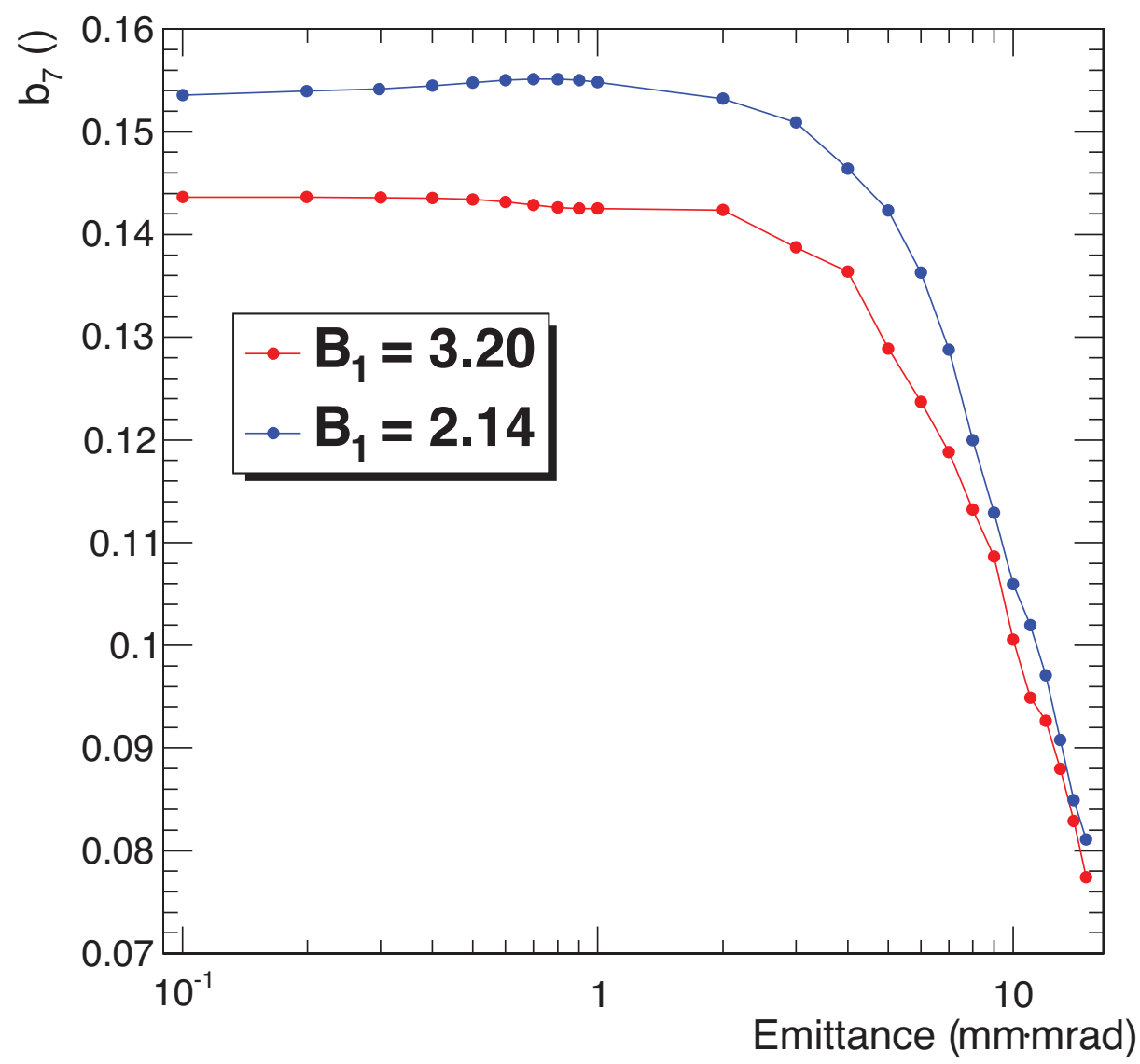

Figure 22: Effects of the emittance on the bunching factor for two different values of $B_{1}$.

The relation between the emittance of the beam and the bunching factor is clearly not linear for both chicane configurations. These two curves can be split into two different parts, with a knee at $\epsilon_{x, y}=4 \mathrm{~mm} \cdot \mathrm{mrad}$. For emittances lower than this value, the bunching factor $b_{7}$ reaches the limit at $14.4 \%$ for $B_{1}=3.20$, and $15.4 \%$ for $B_{1}=2.14$. In this range of values, the second chicane configuration is more appropriate to obtain a high bunching factor. For horizontal and vertical emittances higher than $4 \mathrm{~mm} \cdot \mathrm{mrad}$, the bunching factor linearly decreases with an increase of the emittance. For both $B_{1}$ solutions, the slope is $0.6 \% /(\mathrm{mm} \cdot \mathrm{mrad})$. 
For $B_{1}=3.20$, the phase space after the second chicane is extracted by the SDDS toolkit from elegant simulations. Results for $\epsilon_{x, y}=6 \mathrm{~mm} \cdot \operatorname{mrad}$ are shown in Fig. 23, and in Fig. 24 for $\epsilon_{x, y}=15 \mathrm{~mm} \cdot \mathrm{mrad}$. The $x$-scale represents the time, which is inverted compared to plots provided by the 1D code. These two plots illustrate the process of washing out the fine structure of the beam by an increase of the emittance. In Fig. 23, energy strips are preserved after the two modulations and the two dispersion of the beam. However when the emittance is increased to $15 \mathrm{~mm} \cdot \mathrm{mrad}$, these energy strips are no longer separated, and overlap each other (Fig. 24).
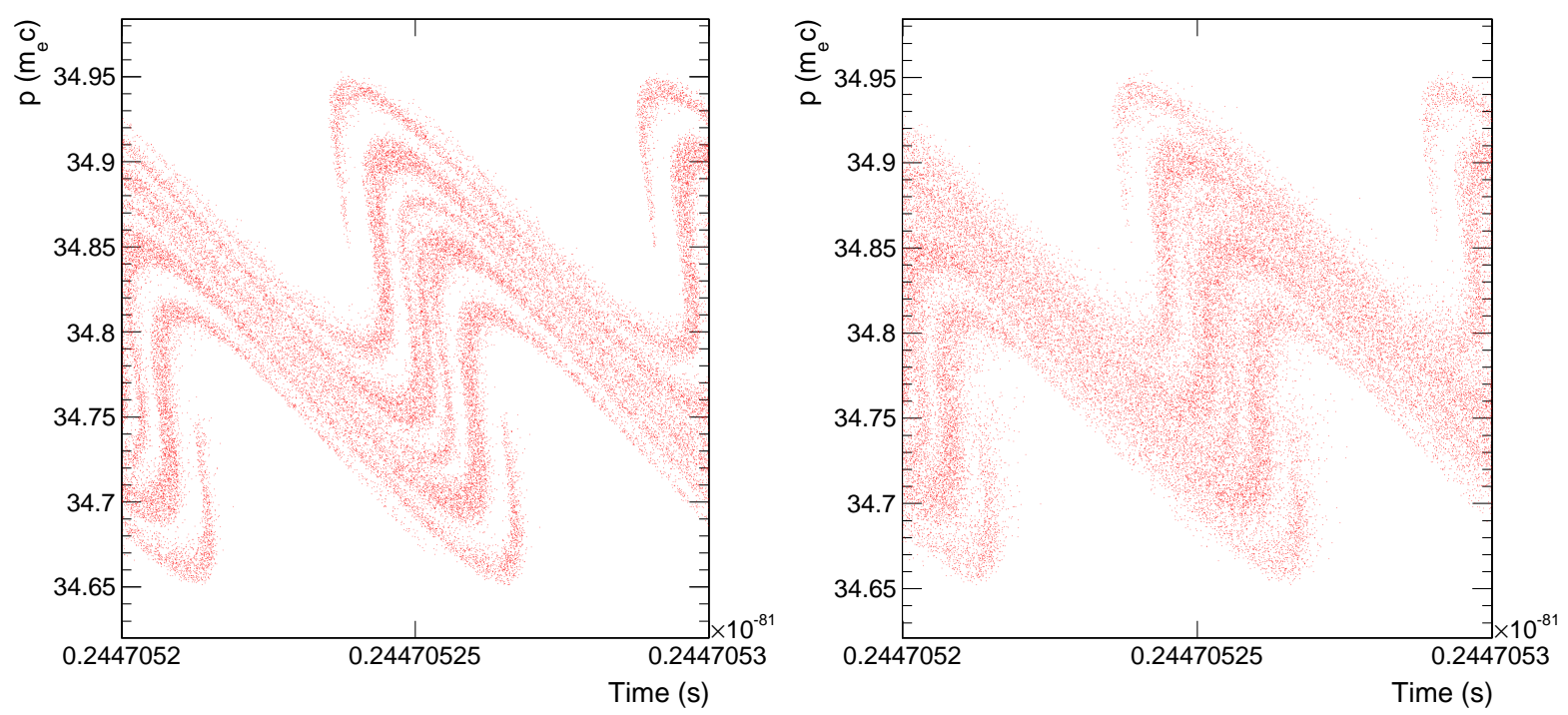

Figure 23: Phase space after the second chi- Figure 24: Phase space after the second chicane for $\epsilon_{x, y}=6 \mathrm{~mm} \cdot \mathrm{mrad}$, and $B_{1}=$ cane for $\epsilon_{x, y}=15 \mathrm{~mm} \cdot \mathrm{mrad}$, and $B_{1}=$ 3.20 . 3.20 .

As a result of these studies, we calculated that the emittance has to be smaller than $8 \mathrm{~mm} \cdot \mathrm{mrad}$ in order to derive a result measured bunching factor of around $11 \%$. 


\subsection{Spontaneous emission in the undulators}

Installation of the Echo-7 experiment was started in Fall 2009 when the chicanes were installed in the beamline. In February 2010, the two first undulators were received and installed. A picture of the second one is shown (Fig. 25). During commissioning, collinearity of electron beam and laser light has to be established. This requires interaction between electrons and the laser. This interaction occurs when the beam and the laser pulses overlap in time and space. By placing an OTR screen ${ }^{9)}$ before and after the undulator, one can check if the electrons and the laser are following the same trajectory inside the undulator.

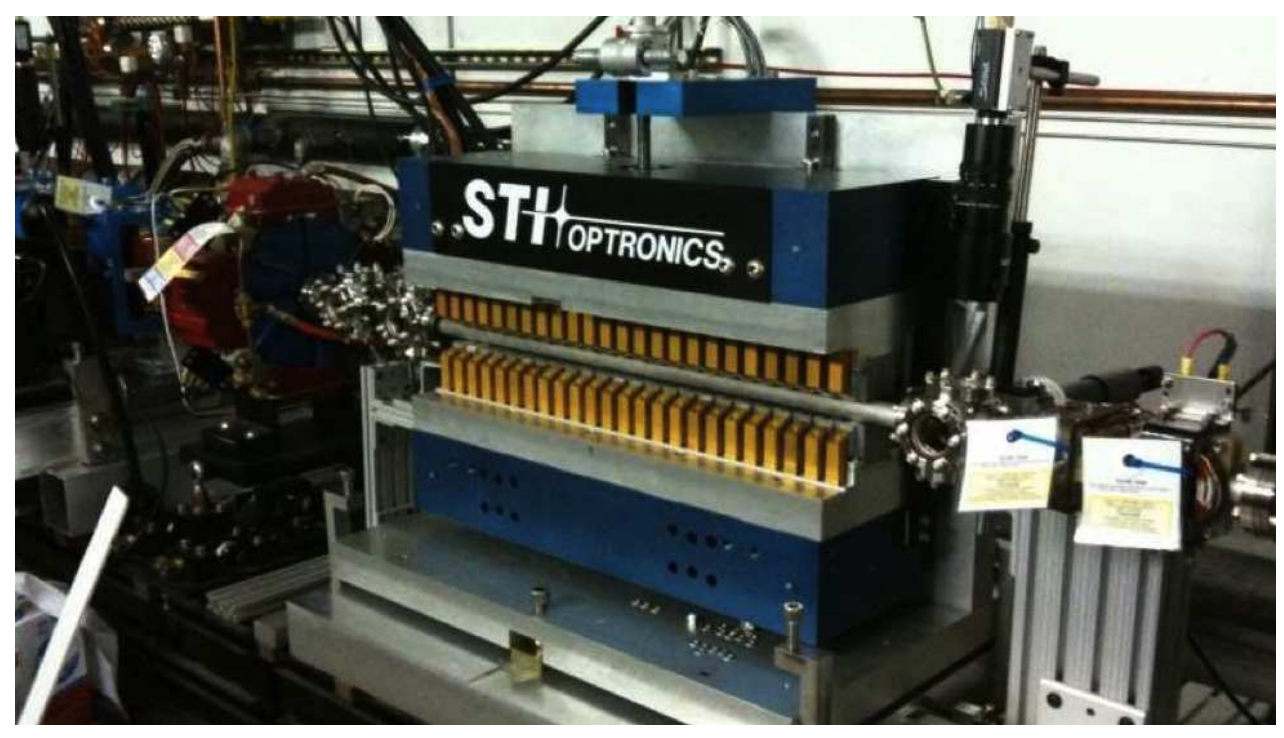

Figure 25: The second undulator after installation in beamline.

For the time overlap, one has to keep in mind that electrons can spontaneously emit light in an undulator, even if there is no coupling to a laser. The first undulator is $33 \mathrm{~cm}$ long, and the second $55 \mathrm{~cm}$, both having ten periods. These characteristics enable microbunching with a high gain, as previously described in Sec. 1.5. Therefore for the time overlap, one measures both laser and spontaneous undulator radiation. In order to be compared with the electron beam, they have to be converted into a time-resolved electrical signal. This is performed by a fast photodiode. The two electrical signals are then sent to an oscilloscope in order to be synchronized. A laser delay line is adjusted until the two signals overlap in time. Photodiodes require a minimum number of photons to produce an electrical signal. Therefore one has to know how many photons are produced by the undulators to choose the appropriate photodiode. The number of photons produced bt OTR cannot be estimated accurately because the quantum efficiency of the screen material is not known.

9) Optical transition radiation (OTR) is emitted when a beam of relativistic particles crosses a material with a different dielectric constant $\epsilon$, in this case a thin aluminum foil. The radiation is detected by a CCD camera. This element is useful to determine the electron beam position. 
The number of photons produced by one electron inside of an undulator can be computed theoretically. The flux of photons spontaneously emitted is confined to a central cone in the zaxis. This can be approximated by a Gaussian profile with a standard deviation $\sigma_{r}=\sqrt{\lambda / L}$. With $\dot{N}_{\gamma}$ the number of photons produced per second, the flux per solid angle $\Omega$ is given by:

$$
\frac{d \dot{N}_{\gamma}}{d \Omega}=\left.\frac{d \dot{N}_{\gamma}}{d \Omega}\right|_{\theta=0} \exp \left(-\frac{\theta^{2}}{2 \sigma_{r}^{2}}\right) .
$$

The angle $\theta$ corresponds to the angle between the $z$-axis and the light received by the observer. By integrating over all angles, one gets (mathematical proofs are demonstrated in [20]):

$$
\dot{N}_{\gamma}=1.43 \times 10^{14} N I_{b} Q_{n}(K),
$$

for a bandwidth $\Delta \omega / \omega=0.1 \%$. Here $N=10$ for the two undulator periods, $K=1.80$ for the first, and $K=2.07$ for the second undulator. The current $I_{b}=1.6 \times 10^{-19}$ A for one electron per second inside of the undulator. The function $Q_{n}(K)$ is the following for the $n^{\text {th }}$ harmonic:

$$
Q_{n}(K)=\frac{1+\frac{K^{2}}{2}}{n} F_{n}(k),
$$

where $F_{n}(K)$ is defined by:

$$
F_{n}(K)=\frac{n^{2} K^{2}}{\left(1+\frac{K^{2}}{2}\right)^{2}}\left(J_{(n+1) / 2}(Y)-J_{(n-1) / 2}(Y)\right)^{2} .
$$

Where $Y=\frac{n K^{2}}{4\left(1+K^{2} / 2\right)}$. The coherent spectral bandwidth is $\Delta \omega / \omega=1 /(n N)$. For the first harmonic, one gets $10 \%$. For the second undulator, due to its configuration, infrared radiation is emitted, therefore one has to look at higher harmonics too. The flux are listed in Tab. 8.

\begin{tabular}{llll}
\hline \hline Undulator & Harmonic & $N_{\gamma}$ for one $e^{-}$ & $N_{\gamma}$ for $40 \mathrm{pC}$ \\
\hline 1 & 1 & 0.019 & $4.8 \times 10^{6}$ \\
2 & 1 & 0.020 & $5.0 \times 10^{6}$ \\
2 & 3 & 0.010 & $2.5 \times 10^{6}$ \\
\hline \hline
\end{tabular}

Table 8: Flux of spontaneous emission for the two undulators.

Finally the chosen photodiode has a $2 \mathrm{GHz}$ bandwidth with a $300 \mathrm{ps}$ rise time. Used with a $2.5 \mathrm{GHz}$ bandwidth oscilloscope, a 50 ps resolution can be achieved. 
At the exit of the undulators, photodiodes are used to acquire light from the combination of undulator spontaneous emission and laser pulses. However this can be contaminated by other light sources as well, which deteriorates the electrical output signal of the diode. A solution is to install a band-pass filter upstream of the OTR screen. Its cutoff frequencies have to be chosen to keep only undulator spontaneous emission and laser pulses. The laser wavelength is known by design, and can be easily measured. However the spectrum of undulator spontaneous emission has to be determined.

On the $z$-axis, the spectrum corresponds to a sinc function defined by: $\operatorname{sinc}(x)=\sin (\pi x) /(\pi x)$, centered at the resonant wavelength. Here, the photodiodes acquires a flux not only on the $z$ axis, but also confined to a central cone. The resonant wavelength depends on the angle $\theta$ from Eq. 20. This fact has to be taken into account to compute the power spectrum of undulator spontaneous emission. Inside the cone, the angular power density is given by [20]:

$$
\frac{d^{2} P}{d \Omega d \omega / \omega} \propto L\left(\frac{N \Delta \omega}{\omega}\right) F_{n}(K) \times \exp \left(-\frac{\theta^{2}}{2 \sigma_{r}^{2}}\right) .
$$

The function $F_{n}(K)$ is given by Eq. 61. When the flux is off-axis, the $Y$ parameter is expressed as:

$$
Y=\frac{n K^{2}}{4 A}
$$

with $A=1+K^{2} /+\theta^{2} \gamma^{2}$. For $\theta=0 \mathrm{mrad}, Y=0.309$, and for $\theta=100 \mathrm{mrad}, Y=0.303$. This justifies the approximation of small $Y$. The difference between the two Bessel functions for $n=1$ in Eq. 61 is then reduced to:

$$
J_{1}(Y)-J_{0}(Y) \approx-1+\frac{Y}{2}+\frac{Y^{2}}{4} .
$$

In Eq. 62, the function $L(N \Delta \omega / \omega)$ is defined by:

$$
L\left(\frac{N \Delta \omega}{\omega}\right)=\frac{\sin ^{2}\left(\frac{N \pi \Delta \omega}{\omega}\right)}{N^{2} \sin ^{2}\left(\frac{\pi \Delta \omega}{\omega}\right)}
$$

The total spectrum is found by integrating Eq. 62 over the cone for different $\omega$. This was performed numerically using the software Maple. Maximum angles $\theta$ are chosen between $1 \mathrm{mrad}$ and $100 \mathrm{mrad}$. Results are presented in Fig. 26 for the first undulator. The absolute value of the intensity is not important, so they are normalized for the different cases in order to compare them. For $x$-scale, the variable $\lambda$ is used instead of $\omega$. The bandwidth of band-pass filters are expressed in wavelength units. 
For the on-axis case, i.e. $\theta=1 \mathrm{mrad}$, one obtains a sinc function as predicted. When the flux is confined to a cone, for $\theta>10 \mathrm{mrad}$, the power peak is shifted to the right. For the first undulator, one has a resonance at $\lambda=821 \mathrm{~nm}$, which is an offset of $3.3 \%$ from the on-axis resonance wavelength $\lambda=795 \mathrm{~nm}$. The shape of the spectrum is also different, it is not a sinc function anymore. For wavelengths higher than the peak value, the tail is a polynomial function. The spectrum bandwidth is no longer $10 \%$ like the on-axis case. It is estimated by calculating numerically the Full Width Half Maximum (FWHM) of the function. One obtains $162 \mathrm{~nm}$ for the first undulator. The new spectrum bandwidth we have to take into account for the choice of the band-pass filter is $19.7 \%$. Same calculations have been computed for the second undulator (Fig. 27). One finds $\lambda=1642 \mathrm{~nm}$ and a FWHM of $320 \mathrm{~nm}$ which gives a bandwidth of $19.5 \%$.
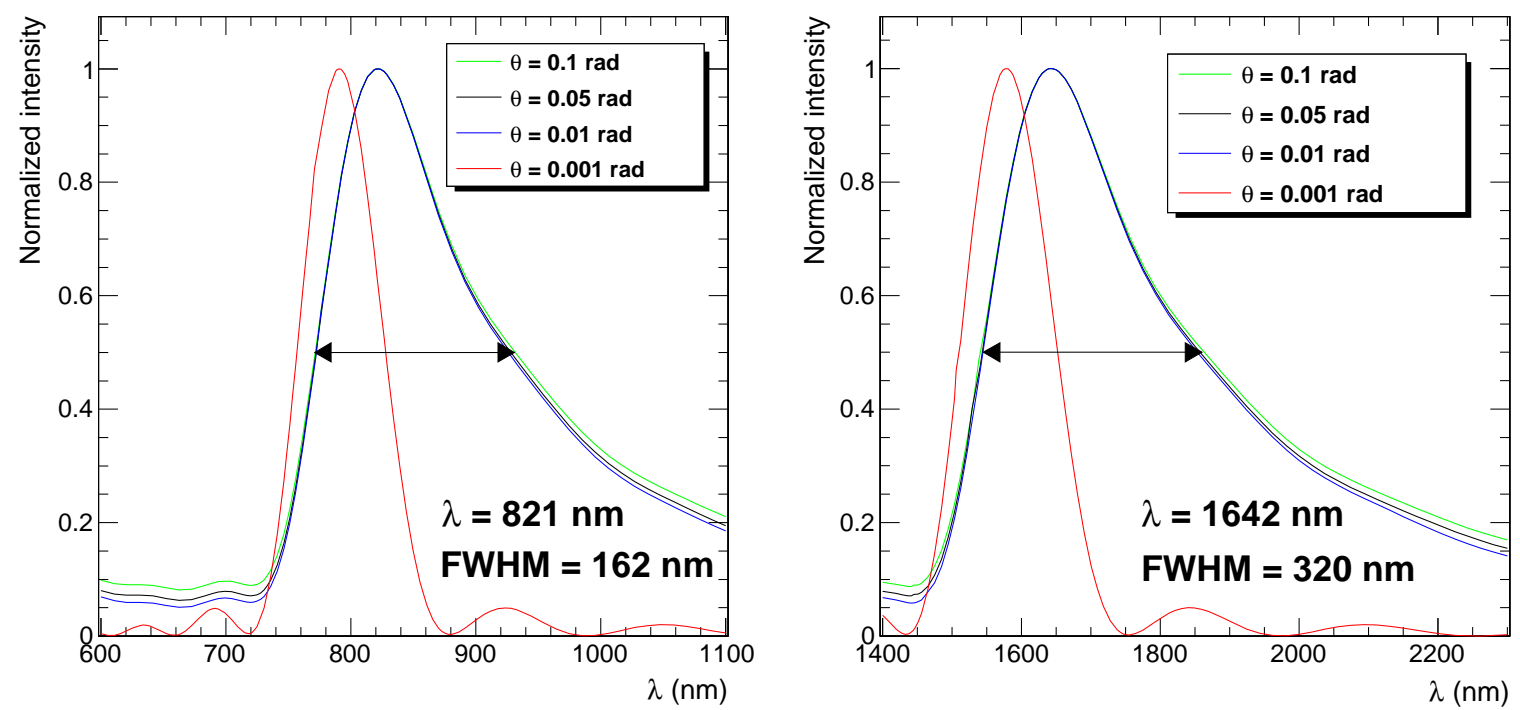

Figure 26: Spontaneous emission spectrum for the first undulator, observed for different cone angles $\theta$.
Figure 27: Spontaneous emission spectrum for the second undulator, observed for different cone angles $\theta$.

Using these results, the more appropriated band-pass filters have been selected. 


\subsection{Second laser phase effect}

For the EEHG effect, the beam energy is modulated separately by two lasers. Physical processes occurring in the undulators are modeled by Eq. 28 for the first one, and by Eq. 39 for the second one. The properties of these two lasers are different, in general they do not have the same power and the same wavelength, resulting in a different modulations of the beam. Another parameter, which influences the bunching factor, is the relative phase difference $\phi$ between the two lasers.

After the first chicane, we have previously seen that horizontal energy strips are created in phase space (Fig. 12). These are directly responsible for the harmonic generation when the energy modulation is converted into a density modulation. In order to obtain higher harmonics, more than the fifth, a large modulation amplitude coupled with a large dispersion is needed. These conditions can be rewritten as a function of the modulation amplitude $A_{1}$ and of the momentum compaction factor $B_{1}$ normalized in energy, as $A_{1} B_{1} \gg 2 \pi$. On the other hand, when the beamline is designed for smaller harmonics, less than the fifth harmonic, $R_{56}$ of the first chicane is reduced. This stems from solutions of Eq. 51. In this case, $A_{1} B_{1} \lesssim 2 \pi$.

For this case, after the first chicane, energy strips are not horizontal anymore. They are of the same order as the first laser wavelength, and the sinusoidal shape in phase space is still visible. When the beam goes through the second undulator, these structures can be canceled or at least their amplitude is reduced by the second laser. This occurs if the two modulations are off phase, such that the peaks of the second modulation corresponds to the valleys of the first one.

Due to technical reasons, this phenomena will be illustrated for the fourth harmonic of the second laser. The same calculations in Sec. 3.1, have been performed to deduce parameters for the two undulators, their lasers, and the two chicanes. Laser parameters are kept unchanged. In order to find $B_{1}$ and $B_{2}$, Eqs. 50 and 51 are solved. There are still an infinite number of solutions for the $B_{1}$ parameter; two of them are listed in Tab. 9. For the fourth harmonic, the condition $A_{1} B_{1} \lesssim 2 \pi$ is fulfilled.

\begin{tabular}{lllll}
\hline \hline & $A_{1}$ & $A_{2}$ & $B_{1}$ & $B_{2}$ \\
\hline Solution 1 & 3 & 4 & 1.34 & 0.93 \\
Solution 2 & 3 & 4 & 2.40 & 0.93 \\
\hline \hline
\end{tabular}

Table 9: Parameters for the fourth harmonic.

Generation of smaller harmonics increases $R_{56}$ for the first chicane, but decreases it for the second one. Solutions close to the $B_{1}$ currently used for the Echo-7 experiment, here $B_{1}=2.14$, are privileged. These avoid large changes in the configuration of the beamline, especially for the magnetic field of the chicanes.

Solution 2 is chosen for the following studies. In order to illustrate the second laser phase effect on the fine structure of the beam, elegant simulations are run. Phase spaces before and after the second modulator are presented in Figs. 28 and 29, for the relative phases $\phi=0$ and $\phi=\pi / 2$. 

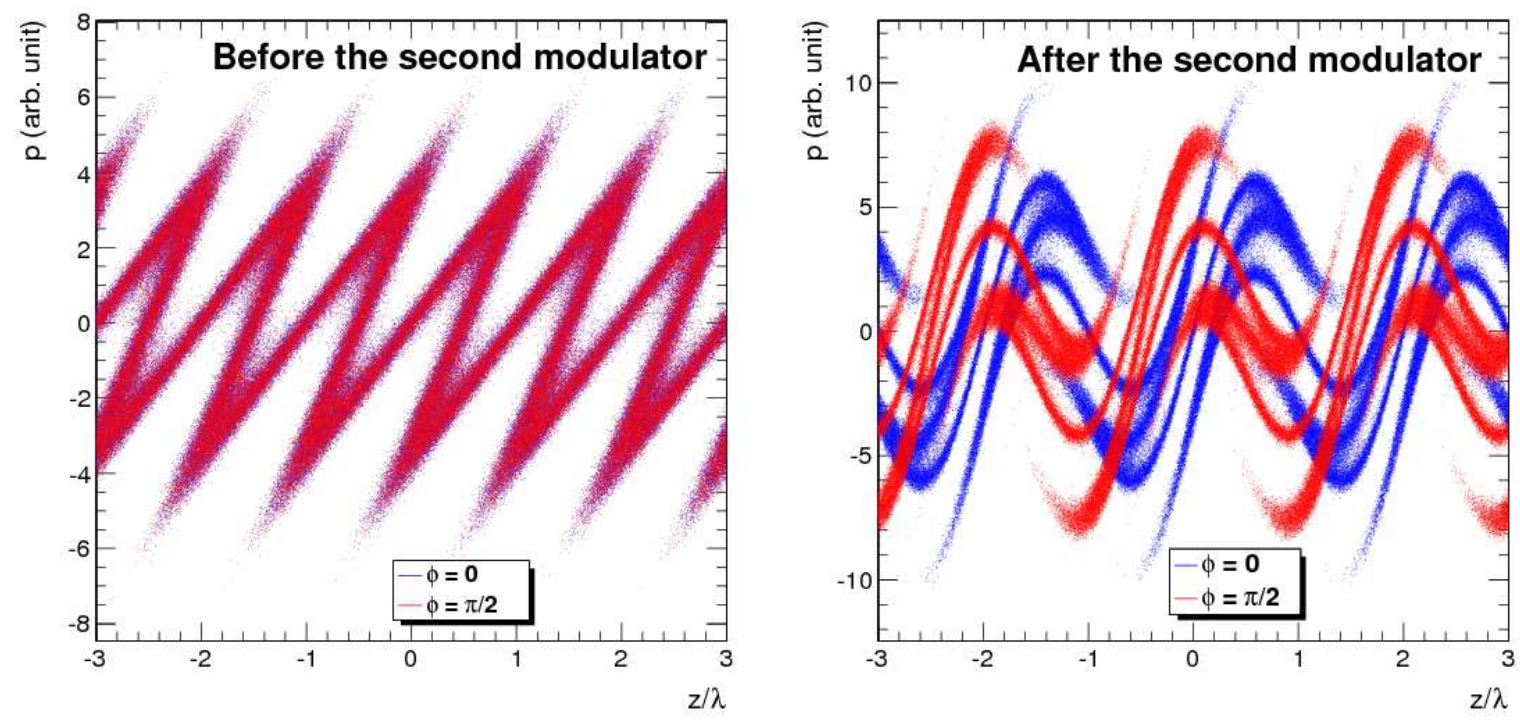

Figure 28: Phase space before the second modulator for $\phi=0$ and $\phi=\pi / 2$.

Figure 29: Phase space after the second modulator for $\phi=0$ and $\phi=\pi / 2$.

Before the second modulator, the two phase spaces overlap. One notices that when $A_{1} B_{1} \lesssim 2 \pi$, the energy strips are no more horizontal, but have an angle of $45^{\circ}$ with the $z$-axis. The energy modulation was not completely sheared by the first chicane. The structure has still a period of the same order of the first laser wavelength.

After the second modulator, the energy of the beam has been modulated in both cases. As expected, when $\phi=0$, the phase undergoes a shift of $+\pi / 2$ compared to $\phi=0$. The $p$-scale is in the same arbitrary units for the two curves, they can then be compared. The maximum amplitude of the modulation for $\phi=\pi / 2$ is 8 , and for $\phi=0$ is 10 . The power of the second laser is the same for these two simulations. The only variable parameter is the phase of the second laser. Therefore the amplitude of each energy modulation depends on this phase, they can be either decreased or increased. The fine structure of the beam being modified, it will affect the bunching factor for the optimized harmonic. This effect is analyzed by doing a scan of the phase.

This phase dependence highlighted by the study of the fourth harmonic is first analyzed in details for the Echo-7 experiment. The same configuration of the beamline listed in Tab. 3 is used. Solutions 2 and 3 give respectively $A_{1} B_{1}=6.42 \sim 2 \pi$ and $A_{1} B_{1}=9.60>2 \pi$. The first solution is close to the condition to have a phase dependence of the bunching factor. Solution 3 should be independent of $\phi$. For these two configurations, 1D simulations are run for different relative phases of the second laser with $\phi \in[0,2 \pi]$. The bunching factor for the seventh harmonic is computed, and results are shown in Fig. 30. 

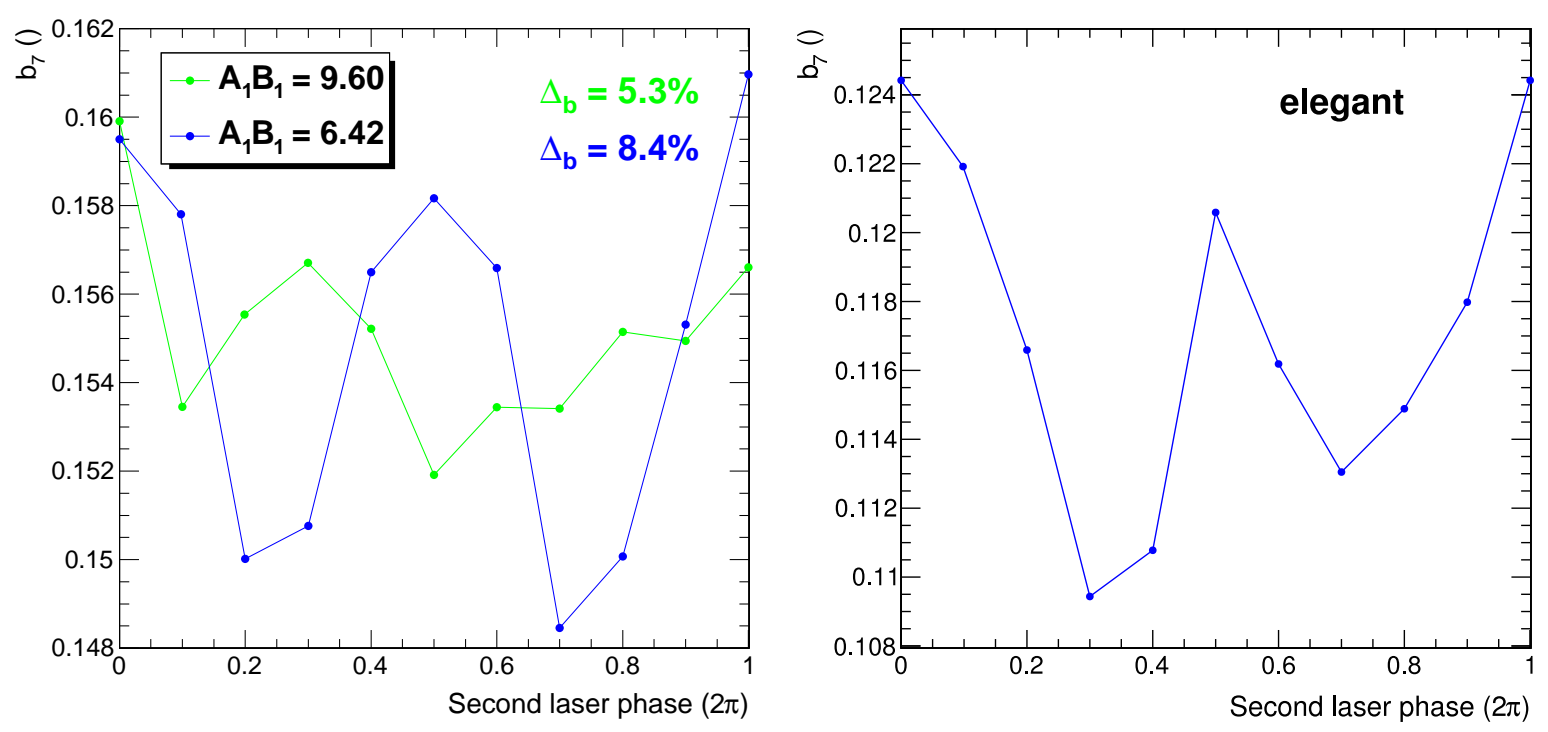

Figure 30: Bunching factor of the sevFigure 31: Bunching factor of the seventh harmonic as a function of the phase of the second laser for two configurations from enth harmonic as a function of the phase of the second laser from elegant simulations. $1 \mathrm{D}$ simulations.

For both cases, the bunching factor of the seventh harmonic $b_{7}$, evolves periodically with the phase of the second laser. It follows a quasi-sinusoidal shape. For $A_{1} B_{1}=9.60$, the amplitude of the oscillations are relatively small, there is only a difference of $5.3 \%$ between the maximum and the minimum of $b_{7}$. However, when $A_{1} B_{1}=6.42$, amplitude of the oscillations is larger, up to $8 \%$ of the initial value. The bunching factor is maximized for $\phi=0$ and $\phi=\pi$; and minimized when $\phi=\pi / 2$ and $\phi=3 \pi / 2$. This periodicity is explained by the ratio between the wavelength of the second and the first laser, which is two.

In order to confirm these results, 6D computer simulations were run with elegant. For $A_{1} B_{1}=6.42$, relationship between the bunching factor and the phase of the second laser is shown in Fig. 31. The same phenomena is observed, $b_{7}$ is maximized when $\phi=0$ and $\phi=\pi$; and minimized when $\phi=\pi / 2$ and $\phi=3 \pi / 2$. Our 1D simulations are benchmarked by the 6D code. Theoretically, the bunching factor for the $k^{\text {th }}$ harmonic $b_{k}$ is given by Eq. 48 . Introducing the second laser phase $\phi$ in this formula gives [21]:

$$
\begin{aligned}
b_{k}= & \mid \exp \left(i m \phi-\frac{\left(-B_{1}+(K m-1) B_{2}\right)^{2}}{2}\right) \times J_{m}\left[-(K m-1) A_{2} B_{2}\right] \\
& \times J_{1}\left[-A_{1}\left(-B_{1}+(K m-1) B_{2}\right)\right] \mid
\end{aligned}
$$

Analytical bunching factors for the seventh harmonic are computed for different phases. Results are shown in Fig. 32. They are compared to the 1D simulation, where the bunching factor for each phase point is the average over five runs, and its error is the rms. These fluctuations are also confirmed by the analytical solution. 

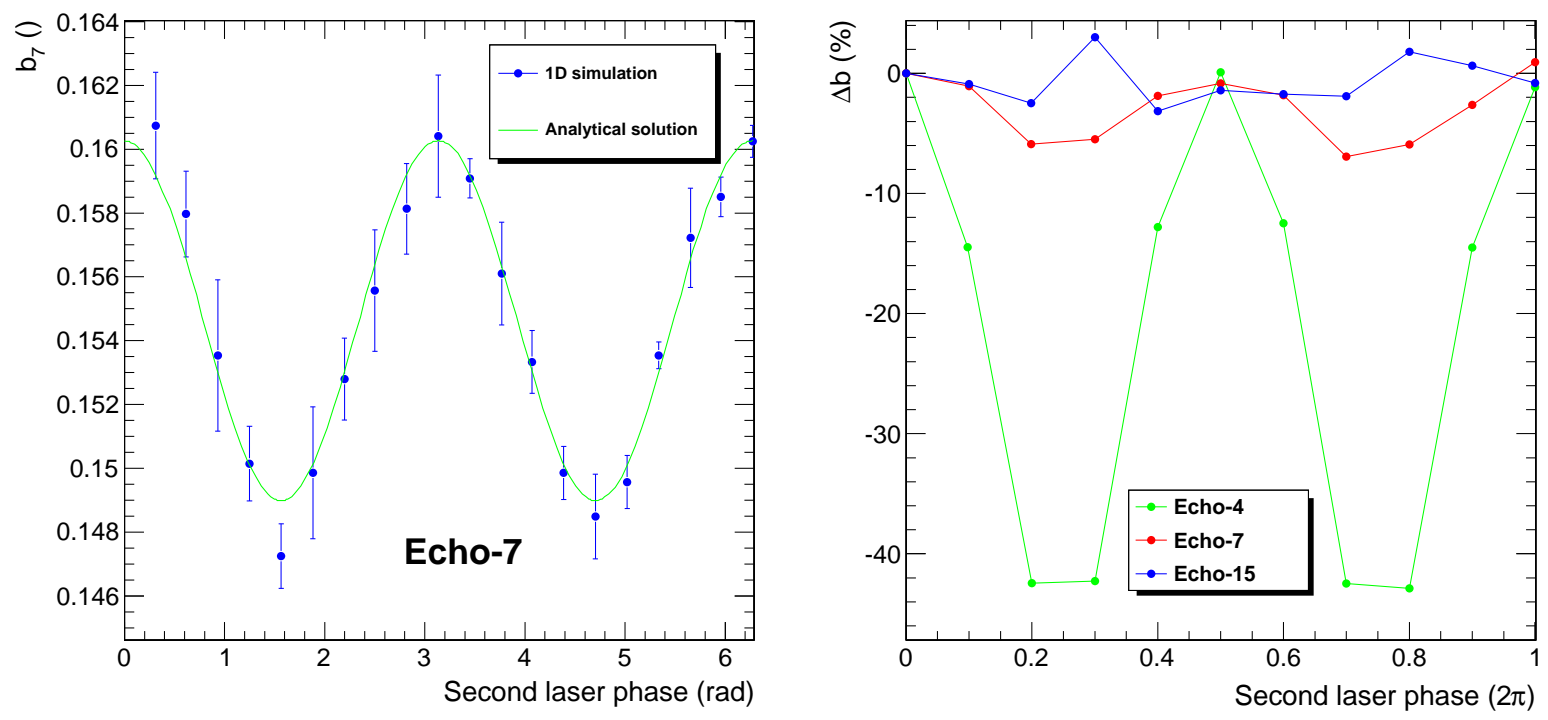

Figure 32: Comparison between the bunching factor calculated from the analytical soFigure 33: Comparison of the phase depenlution, and from 1D simulations. dence between Echo-4, Echo-7 and Echo-15.

A consequence of this effect is the choice of the harmonic when designing a proof-of-principle experiment for the EEHG technique. A comparison of the phase dependence between Echo-4, Echo-7 and Echo-15 is shown in Fig. 33. The vertical scale represents the relative difference of the resonant bunching factor $\Delta b$ with respect to $b(\phi=0)$. Configuration parameters of the beamline for these three different cases are listed in Tab. 10.

\begin{tabular}{lllll}
\hline \hline Harmonic & $A_{1}$ & $A_{2}$ & $B_{1}$ & $B_{2}$ \\
\hline $4^{\text {th }}$ & 3 & 4 & 1.34 & 0.93 \\
$7^{\text {th }}$ & 3 & 4 & 2.14 & 0.76 \\
$15^{\text {th }}$ & 3 & 4 & 4.24 & 0.64 \\
\hline \hline
\end{tabular}

Table 10: Parameters for the Echo-7 experiment, for different harmonics.

As expected, 1D simulations show that for low harmonics, oscillations are much larger than for higher ones. For Echo-4, the difference between $b_{4}$ at $\phi=0$ and at $\phi=\pi / 2$ is more than $40 \%$. It is only $6 \%$ for $b_{7}$ in Echo-7, and less than $4 \%$ in $b_{15}$ for Echo- 15 .

For the Echo-7 experiment at the NLCTA, this phase effect should not be a major factor in achieving an adequate bunching factor. However, at lower harmonics, $\phi$ has to be well defined in order to achieve the highest possible bunching factor. 


\subsection{Measurement of the jitter in the RF structures}

At the NLCTA, the beam is produced by a RF photocathode gun. A laser heats the cathode in order to extract electrons. These are are then accelerated up to the energy of $60 \mathrm{MeV}$ by a Xband RF structure ${ }^{10}$ ), called station 0. Different experiments at the NLCTA uses this beam. For Echo-7, the beam is boosted to $120 \mathrm{MeV}$ by another X-band RF structure, here called station 2.

A difficult challenge for the Echo-7 experiment is the time overlapping between the beam and the lasers in the two undulators. For this task, one needs to know accurately the relative timing jitter between station 0 and station 2. One has to look at their phase difference. A so called phase mixer is used. It is composed of three different ports: the radio frequency (RF) port, the local oscillator port (LO), and the intermediate frequency (IF) port. The phase mixer multiplies the RF signal by the LO signal. Considering $V_{\mathrm{RF}}=A_{1} \cos \left(\omega_{1} t+\phi_{1}\right)$, and $V_{\mathrm{LO}}=A_{2} \cos \left(\omega_{2} t+\phi_{2}\right)$, the voltage at the IF port is:

$$
V_{\mathrm{IF}}=G \frac{A_{1} A_{2}}{2}\left[\cos \left(\omega_{1}+\omega_{2}\right) t+\left(\phi_{1}+\phi_{2}\right)\right)+\cos \left(\left(\omega_{1}-\omega_{2}\right) t+\left(\phi_{1}-\phi_{2}\right)\right],
$$

with $G$ the gain. In our case, signal for the radio frequency (RF) port comes from station 2 and from station 0 for the local oscillator (LO) port. For both stations, angular frequencies are the same, $\omega_{1}=\omega_{2}$, therefore Eq. (67) becomes:

$$
\left.V_{\mathrm{IF}}=G \frac{A_{1} A_{2}}{2}\left[\cos \left(2 \omega_{1}\right) t+\left(\phi_{1}+\phi_{2}\right)\right)+\cos \left(\phi_{1}-\phi_{2}\right)\right] .
$$

The $\omega_{1}+\omega_{2}$ term is on the order of $22 \mathrm{GHz}$. A low-pass filter with a $1 \mathrm{GHz}$ cutoff frequency is installed after the phase mixer in order to remove the high frequency term . Therefore, Eq. (68) is now:

$$
V_{\mathrm{IF}}=G \frac{A_{1} A_{2}}{2} \cos \left(\phi_{1}-\phi_{2}\right)
$$

In the linear regime, $\phi_{1}-\phi_{2}$ is close to $\pi / 2$. A trombone phase shifter ${ }^{11)}$ is use before the RF port to change $\phi_{1}$. When $\phi_{1} \approx \phi_{2}+\pi / 2$, Eq. (69) can be expressed as:

$$
V_{\mathrm{IF}} \approx G \frac{A_{1} A_{2}}{2}\left(\phi_{1}-\phi_{2}\right) .
$$

10) In this regime, RF structures are energized by klystrons at a frequency between 8 and $12 \mathrm{GHz}$.

11) A trombone phase shifter is a mechanical analog phase shifter, changing the phase with a variable length transmission line. 
The resulting IF signal is proportional to the phase difference between the two stations. The voltage $V_{\mathrm{IF}}$ is read by an oscilloscope using a trigger locked to the RF generating source. A scheme of the connections is shown in Fig. 34, and a photograph of the entire set-up is shown in Fig. 35. The trombone phase shifter is blue.

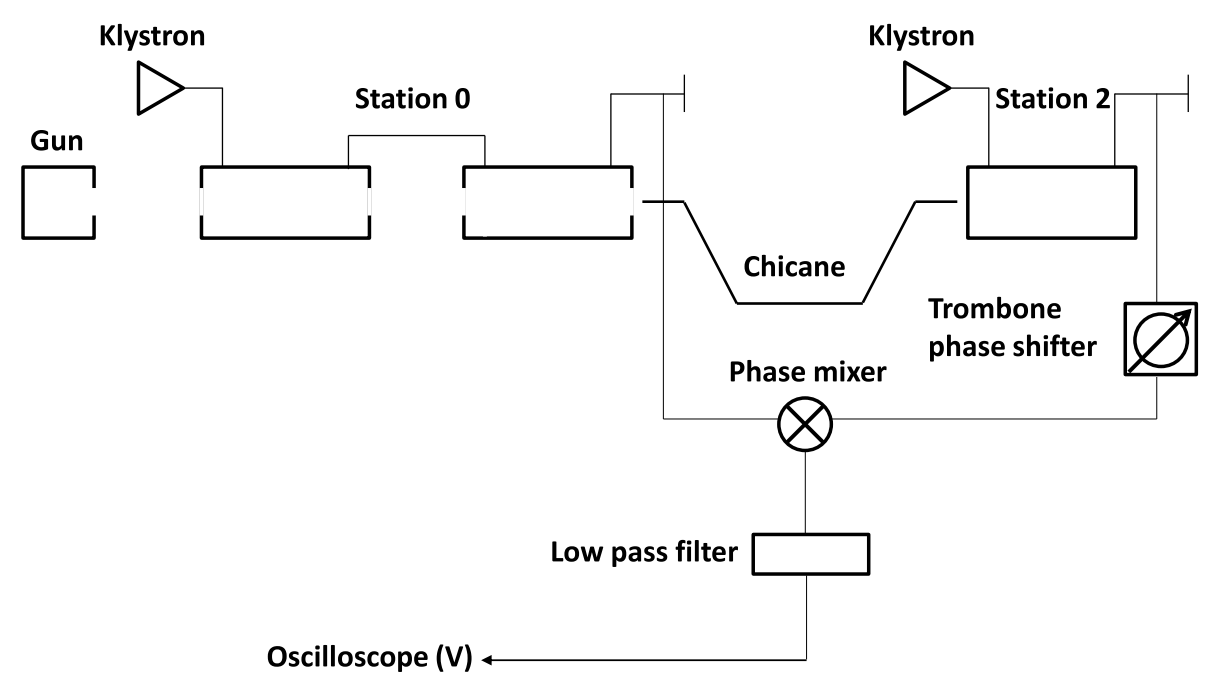

Figure 34: Sktech of the connections of the RF two stations, phase shifter and the phase mixer.
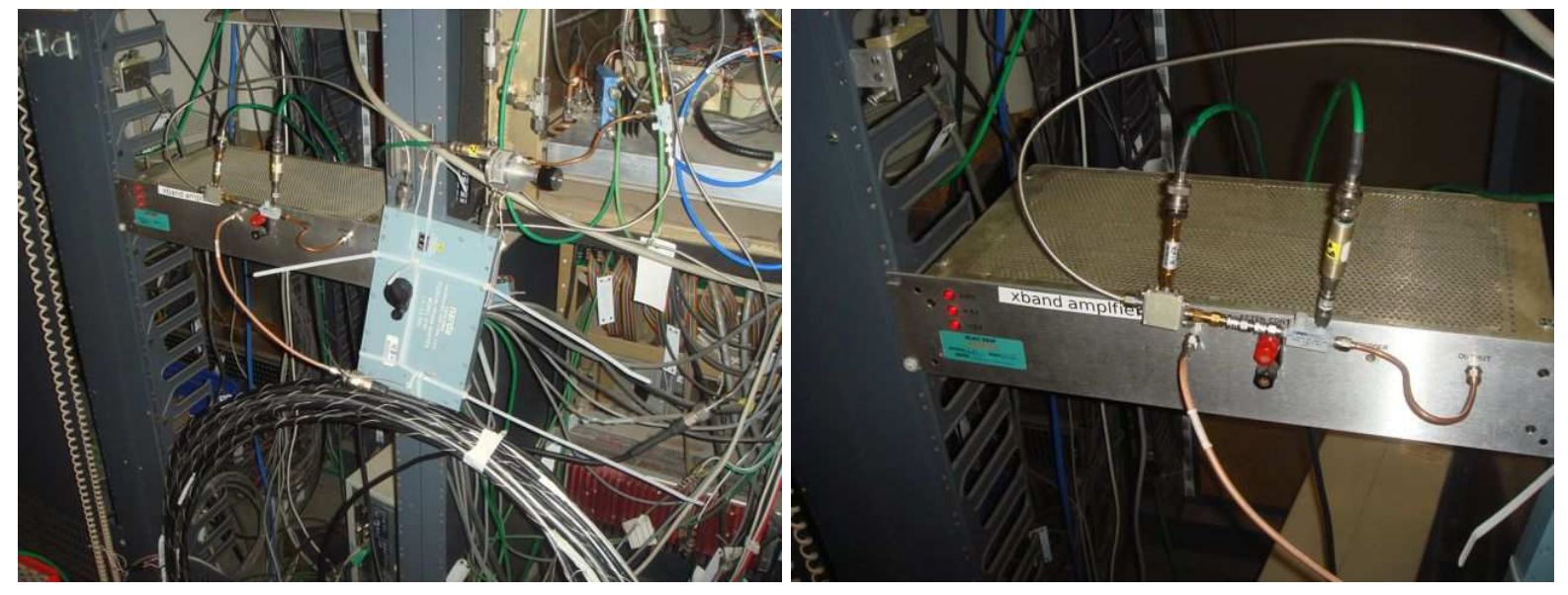

Figure 35: Photograph of the phase shifter before the RF port.
Figure 36: Photograph of the phase mixer with LO signal from station 0 , and RF signal from station 2 . 
The phase shifter has to be calibrated in order to compute the $G \times A_{1} A_{2} / 2$ factor of Eq. (70). This is done by performing a phase scan over $2 \pi$ with the shifter and measuring the change in the voltage. Here the range of the phase shifter changes from $0^{\circ}$ to $360^{\circ}$ of $11.424 \mathrm{GHz}$. Data from the oscilloscope is retrieved by LabView code, and analyzed with the Root data analysis framework. More details of the phase mixer are shown in Fig. 36.

The calibration result is shown in Fig. 37. The symbol $U$ represents the voltage read at the oscilloscope. Data is fitted by a sine function. In the linear domain, when $U \approx 0$ and $U^{\prime}$ is maximized, $1 \mathrm{mV}$ represents $0.65^{\circ}$. For X-band, $1^{\circ}$ corresponds to 0.25 ps of time delay. Therefore, $1 \mathrm{mV}$ on the oscilloscope is equal to $0.16 \mathrm{ps}$.
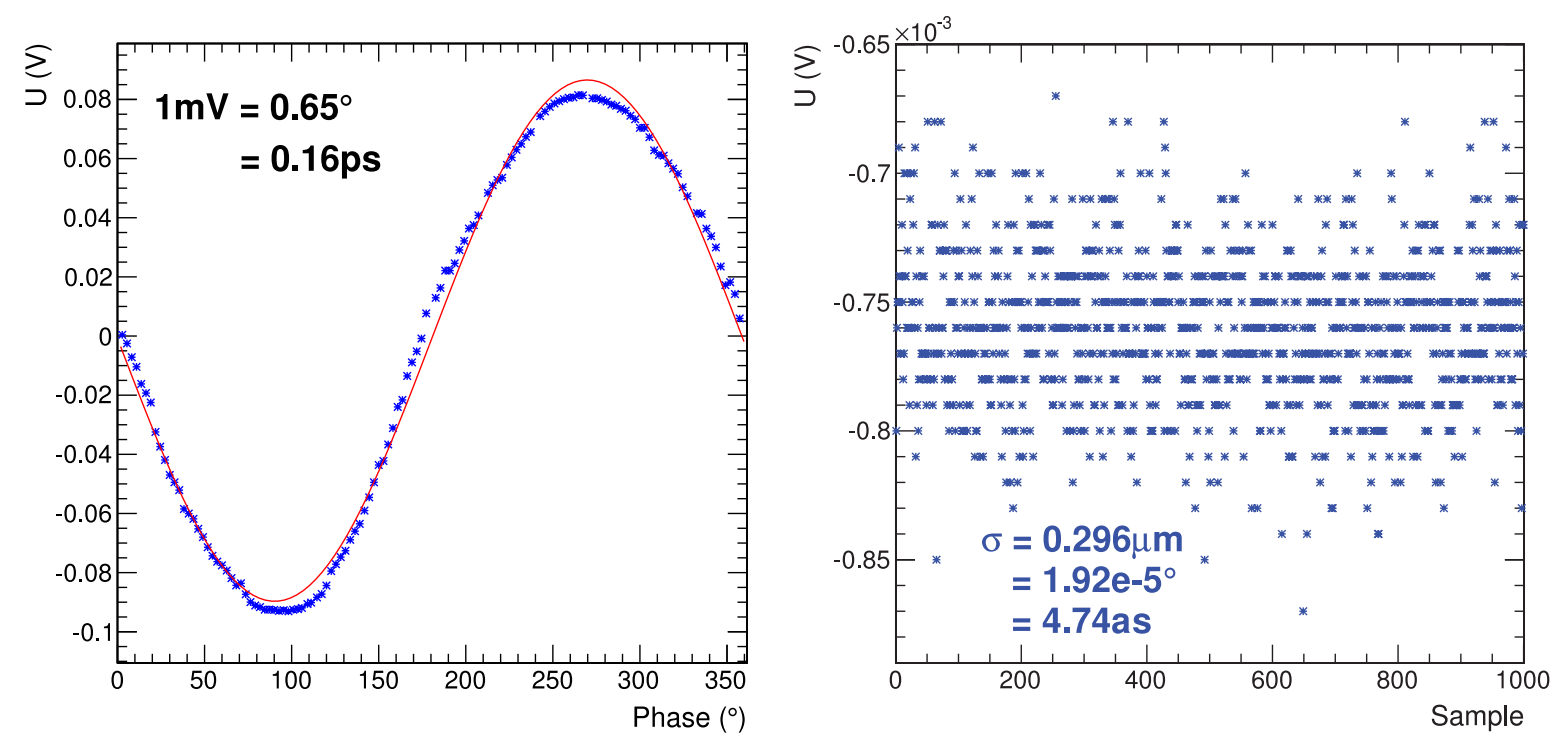

Figure 37: Calibration of the phase shifter in Figure 38: Background noise from the difvoltage by scanning the phase. ferent electrical components.

The background noise of these different electrical components was measured to determine the resolution. For this task, RF amplitudes for station 0 and station 2 are set to zero, but with modulators were still pulsing. Data are acquired, see Fig. 38. The rms of this noise level is only $0.296 \mu \mathrm{V}$, which corresponds to a time delay of 4.74 as. This background noise is negligible compared to the signal. 
In order to measure the phase jitter between station 0 and station 2, data are acquired during one minute intervals while both stations were turned on (Fig. 39). The deviation is $\sigma=1.35 \mathrm{mV}$, it corresponds to a timing jitter of 0.34 ps.
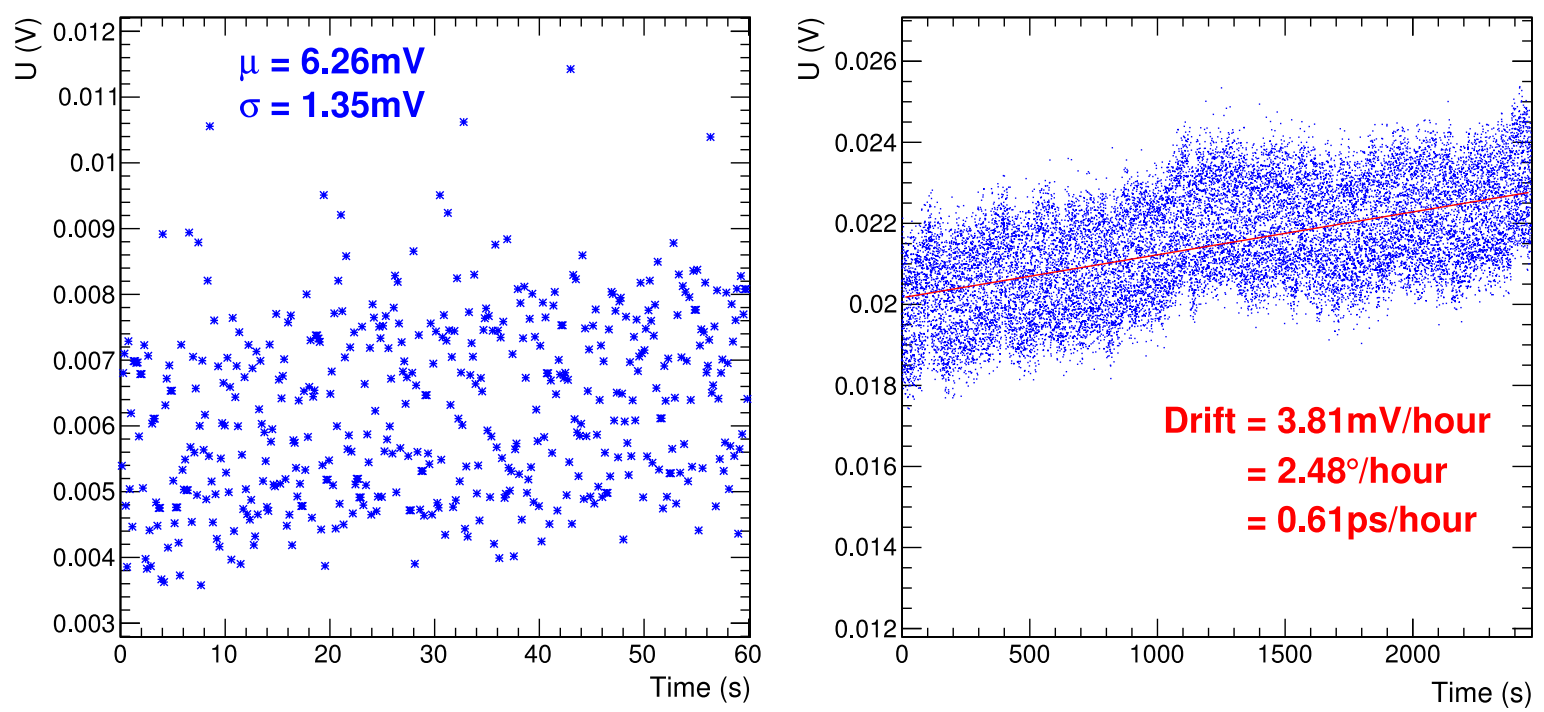

Figure 39: Measurement of the timing jitter Figure 40: Measurement of the drift between between station 0 and station 2 . station 0 and station 2.

Finally, the phase drift between the two stations is measured by acquiring data over a full hour (Fig. 40). A linear regression is performed on the points in order to deduce its trend. The timing drift is $0.61 \mathrm{ps} / \mathrm{h}$. 


\section{Status of the Echo-7 experiment}

\subsection{Current results obtained}

Installation of the Echo-7 experiment started Fall 2009 at the NLCTA, after the design and development stages. End of March 2010, the three chicanes, the two modulators and the radiator were in place on the beamline. Commissioning of the experiment began in April 2010; first results are now described here.

The initial goal of this EEHG proof-of-principle experiment is to produce the fourth harmonic of the second laser. For a low harmonic, it is easier to preserve the fine structure of the beam. In order to reduce the bend angle errors of each chicane, the four bend magnets are powered by the same power supplies. In addition, trim windings are used for equalizing the magnetic fields. An emittance of $5 \mathrm{~mm} \cdot \mathrm{mrad}$ has been achieved at the beginning of the Echo-7 beamline. Position jitter of both the electron beam and the lasers has been measured and is smaller than the rms size of the beam and lasers. Therefore the spatial overlapping of the lasers and the beam in the two undulators was observed [15].

To measure the timing overlap, a fast scanning delay stage was used to synchronize the electron bunches with the laser pulses. Due to microbunching in the first modulator and chicane as described in Sec. 1.5, an enhancement of the COTR radiation was observed downstream of the first chicane on April 22. This verifies the laser-beam interaction; for the second undulator, it was observed on May 8. The COTR radiation as a function of the laser timing for the second undulator is shown in Fig. 41. At a time delay of $67.5 \mathrm{ps}$, the signal is increased.

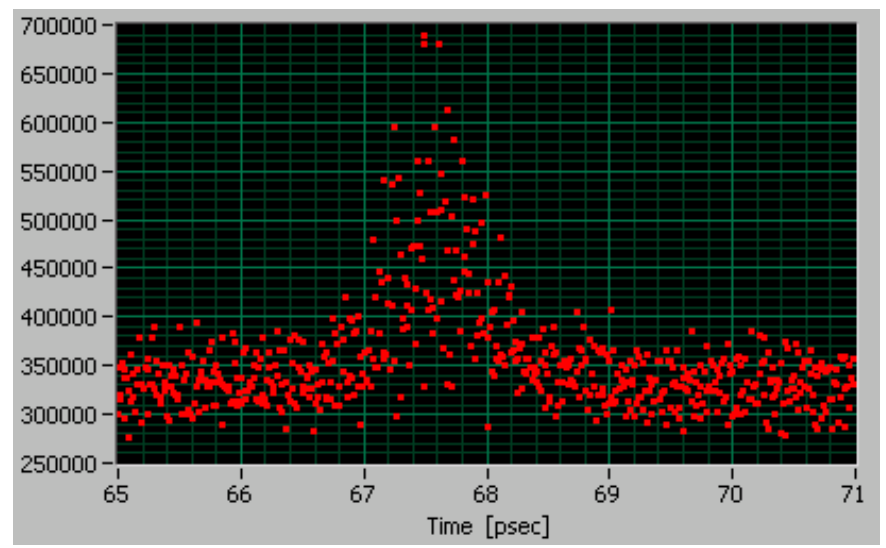

Figure 41: Laser-beam interaction in the second undulator enhances the COTR signal.

At the exit of the radiator, when the beam had interacted with the lasers in each undulator, coherent undulator radiation was observed. A CCD camera acquired the signal. In order to know if the fourth harmonic of the second laser was generated, a band-pass filter with a frequency of $395 \pm 10 \mathrm{~nm}$ was installed. A signal was observed, confirming that the fourth harmonic was generated.

Due to the small energy spread of the beam and the low harmonic number chosen, the signal can stem not only from the EEHG but also from the HGHG effect. Some additional analysis had to be performed to distinguish these two possible contributions. These are ongoing and not part of this thesis. 


\subsection{A possible outlook: Echo-15}

An advantage of the EEHG technique over the HGHG method is the possibility to produce higher harmonics more efficiently. Currently, investigation of the fourth and fifth harmonics is under way. To study the seventh harmonic and possibly the fifteenth, the radiator needs to be retuned, which will happen at a later stage. For the fifteenth, the signal detection has to be upgraded to a UV CCD camera. The same calculations as in Sec. 3.1 were performed in order to compute the configuration parameters of the chicanes and the undulators. Different solutions for $B_{1}$ can give this resonant harmonic (Fig. 42). In Sec. 3.5, the solution for $B_{1}=4.24$ was chosen for the second laser phase effect studies. The other parameters are presented in Tab. 10 in Sec. 3.5.
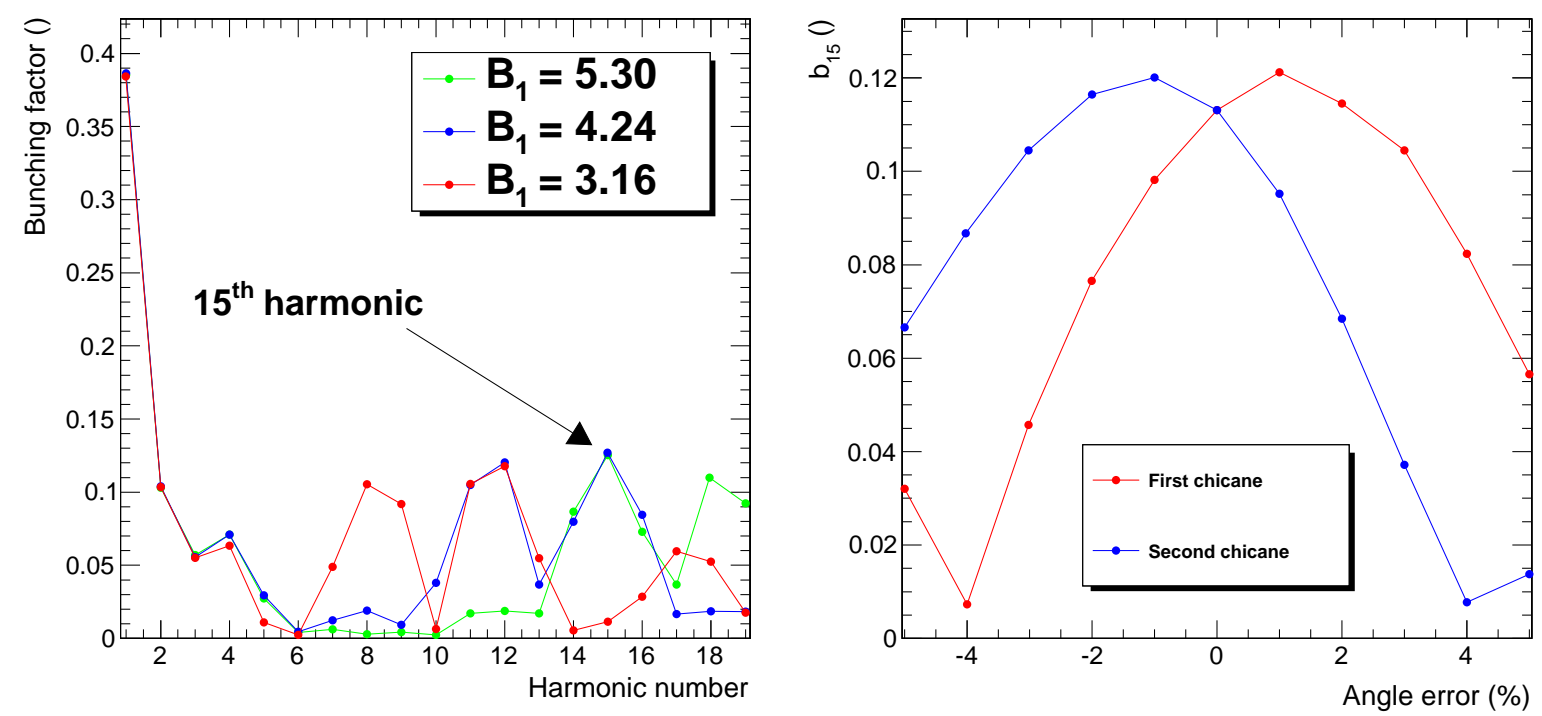

Figure 42: Bunching factor for different $B_{1}$ solutions.

Figure 43: Optimization of the bend angles of the chicanes from elegant simulations.

This solution has been optimized at first order for the bend angles of the two chicanes (Fig. 43). From elegant simulations, the maximum bunching factor achievable for the fifteenth harmonic is $12 \%$. A second order optimization has been made using the Newton's algorithm. Results are presented in Fig. 44. Optimization of the initial parameters do not converge. This may stem from the presence of a local minimum. When the recursive algorithm starts from the the maximum angles found in Fig. 43, the bunching factor converges to $12.3 \%$, with the following bend angles $\theta_{1}=94 \mathrm{mrad}$ and $\theta_{2}=35 \mathrm{mrad}$. 

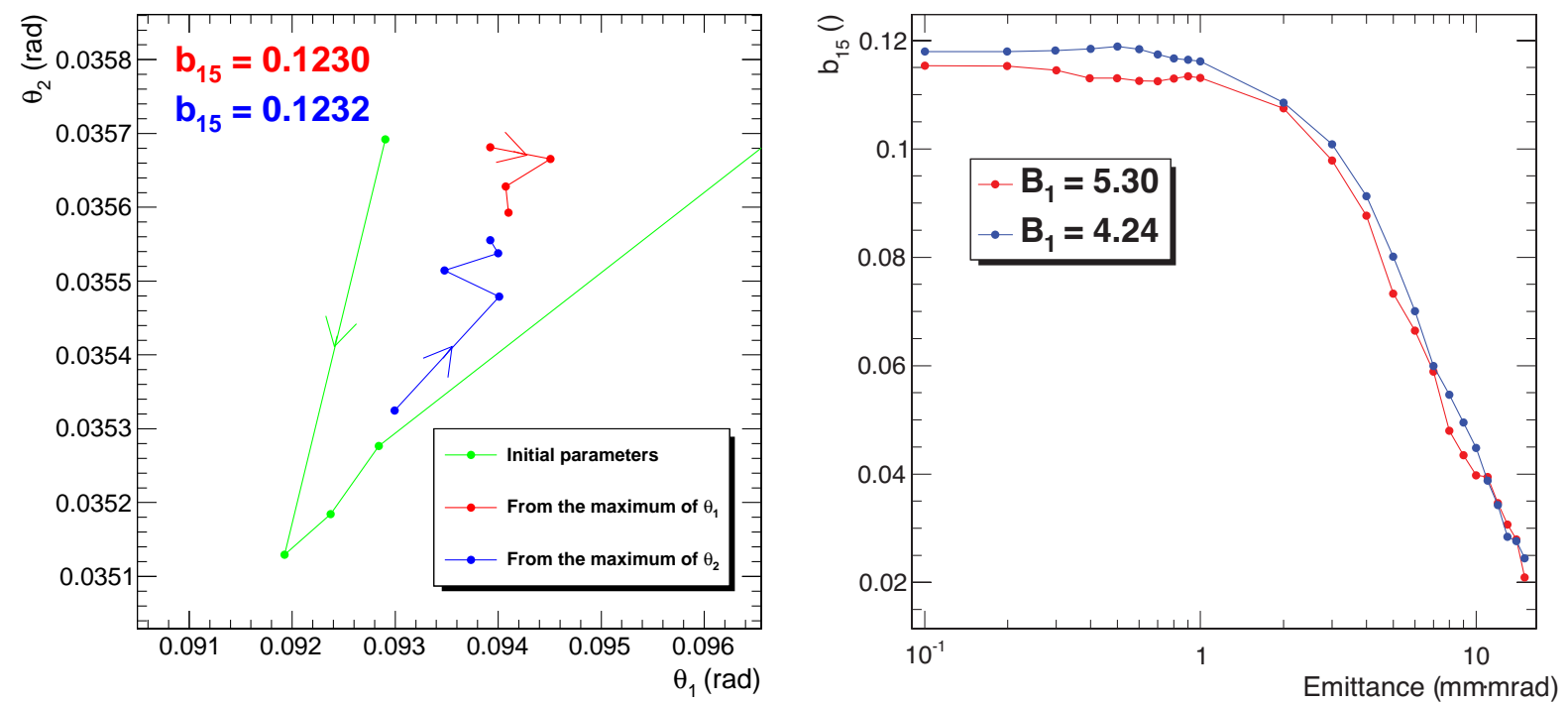

Figure 44: Optimization of the bend angles

Figure 45: Dependence of the bunching facof the chicanes at second order with the Newton's algorithm. tor on the emittance for the fifteenth harmonic.

The effect of the emittance on the bunching factor has been studied also for the $B_{1}=5.30$ solution. For the seventh harmonic, there was a knee at $\epsilon_{x, y}=4 \mathrm{~mm} \cdot \mathrm{mrad}$; for the fifteenth harmonic, the emittance decrease occurs at $\epsilon_{x, y}=2 \mathrm{~mm} \cdot \operatorname{mrad}$ (Fig. 45). A challenge will be to achieve this emittance with the NLCTA beamline, the current emittance in both planes being $5 \mathrm{~mm} \cdot \mathrm{mrad}$. 


\section{Conclusion}

The generation of light at high harmonics by the EEHG method has been proposed in 2009 . This method needs two separate stages where a beam is modulated by a laser in an undulator and sent to a chicane to generate density modulations of the beam. A proof-of-principle experiment has been established at the NLCTA in order to demonstrate this mechanism for the seventh harmonic. It is called Echo-7 and is currently in the commissioning stage.

Following the theoretical description, the necessary parameters of the two undulators and the two chicanes have been computed in order to design the experiment at this chosen harmonics. For the first chicane, the bend angle has been optimized up to the second order using 6D computer simulations, in order to obtain the maximum bunching factor $b_{7}$. Two important factors which have a negative effect on the bunching factor have been studied in detail: the emittance of the beam and the phase of the second laser with respect to the first. Values for both of these parameters have been determined to maximize $b_{7}$. Finally, the timing overlap of the beam with the laser in each undulator is a challenge for this experiment. In order to manage the laser-beam interaction, the spontaneous emission in the undulators has been measured, as well as the timing jitter between the two RF structures used to accelerate the beam up to $120 \mathrm{MeV}$.

First data has been acquired and analyzed. An EEHG signal has been observed for the fourth harmonic of the second laser. However more studies are needed in order to understand if this signal comes from EEHG or HGHG. Upgrades will be necessary for the Echo-7 beamline to overcome these challenges. For instance, one idea is to increase the laser pulse length in order to mitigate the timing jitter. 


\section{Acknowledgments}

I thank Prof. Leonid Rivkin who gave me the opportunity to realize my master's project at SLAC. I want to thank Prof. Tor Raubenheimer and Carsten Hast who welcomed me at the NLCTA and made everything possible to help me to conduct my research for the Echo-7 experiment. I want to thank Janice, Doug, Keith, Mike and Stephen who created a very good working environment. Finally, thanks to Dao Xiang, Gennady Stupakov and Eric Colby for their physics support on my master's thesis. 


\section{A Electron-laser interaction}

In the following section, the interaction between a laser and an electron beam inside an undulator is described [22]. The relative transverse and longitudinal velocities of an electron are described by:

$$
\begin{aligned}
& \beta_{x}=-\frac{K}{\gamma} \sin \left(k_{u} z\right) \\
& \beta_{z}=1-\frac{1}{2 \gamma^{2}}\left(1+\frac{K^{2}}{2}\right)+\frac{K^{2}}{4 \gamma^{2}} \cos \left(2 k_{u} z\right),
\end{aligned}
$$

where $k_{u}=2 \pi / \lambda_{u}$. By integrating Eq. (71a) over $z$, and Eq. (71b) over $t=z / c$, one obtains the following equations of motion:

$$
\begin{aligned}
x(z) & =x_{0}+\frac{K}{k_{u} \gamma} \cos \left(k_{u} z\right) \\
z(t) & \approx c t-\frac{1}{2 \gamma^{2}}\left(1+\frac{K^{2}}{2}\right) c t+\frac{K^{2}}{8 k_{u} \gamma^{2}} \sin \left(2 k_{u} z\right) .
\end{aligned}
$$

Inside of the undulator, an energy transfer will take place between the electron and the laser. With $E_{x}$ the polarized laser field in the horizontal plane, the relativistic factor of the electron will evolve as:

$$
\frac{\gamma}{d t}=\frac{e}{m c} E_{x} \beta_{x}
$$

For the laser field, as an example, we choose the Hermite-Gaussian TEM $_{10}$ mode [23]:

$$
E_{x}=\frac{E_{0}}{1+\left(z / z_{0}\right)^{2}} \frac{2 \sqrt{2} x}{\omega_{0}} \sin (k(c-t)+\psi) \times \exp \left[-\frac{(z / c-t+s / c)^{2}}{4 \sigma_{\tau}^{2}}\right],
$$

with $E_{0}$ the field amplitude, $k$ the wave vector, $z_{0}=k \omega_{0}^{2} / 2$ the Rayleigh length, $\omega_{0}$ the waist size in the center of the undulator, $s$ the electron coordinate in the bunch, $\sigma_{\tau}$ the rms width of the laser pulse intensity. The phase of the wave is defined by:

$$
\psi=\psi_{0}-2 \tan ^{-1}\left(\frac{z}{z_{0}}\right)
$$

Here $\psi_{0}=k s$ is the phase of the wave at the entrance of the undulator, and $R=\left(z^{2}+z_{0}^{2}\right) / z$. For Eq. (74) and Eq. (75), the assumption of $\sigma_{x, y} \ll \omega_{0}$ has been made. Then, by combining Eq. (71), Eq. (73) and Eq. (74), one gets:

$$
\begin{aligned}
\frac{d \gamma}{d t}= & \frac{e E_{0} K}{m c \gamma\left(1+\left(z / z_{0}\right)^{2}\right)} \frac{2 \sqrt{2}}{\omega_{0}} \times \sin \left(k_{u} z\right) \sin (k(z-c t)+\psi) \\
& \times \exp \left[-\frac{(z / c-t+s / c)^{2}}{4 \sigma_{\tau}^{2}}\right]\left(x_{0}+\frac{K}{k_{u} \gamma} \cos \left(k_{u} z\right)\right) .
\end{aligned}
$$

The third term can be simplified using the resonant condition

$$
\gamma_{r}^{2}=\frac{k}{2 k_{u}}\left(1+\frac{K^{2}}{2}\right)
$$


and Eq. (72), one gets:

$$
k(z-c t)=-k_{u} c t \frac{\gamma_{r}^{2}}{\gamma^{2}}-\frac{\xi}{2} \sin \left(2 k_{u} z\right),
$$

where $\xi=K^{2} /\left(2+K^{2}\right)$. Rewritten Eq. (78) with first kind Bessel functions results in:

$$
2 \sin \left(k_{u} z\right) \sin (k(z-c)+\psi) \approx\left(J_{0}\left(\frac{\xi}{2}\right)-J_{1}\left(\frac{\xi}{2}\right)\right) \cos \left[k_{u} c t\left(1-\left(\frac{\gamma}{\gamma_{r}}\right)^{2}\right)+\psi\right]
$$

with $J_{0}$ and $J_{1}$ the Bessel functions at the zeroth and the first order. By averaging Eq. (76) over one undulator period, one obtains:

$$
\begin{aligned}
\left\langle\frac{d \gamma}{d \hat{z}}\right\rangle= & \frac{2 \sqrt{2} x_{0}}{w_{0}} \frac{e E_{0} K L_{u}}{2 m c^{2} \gamma}\{J J\} \frac{\cos \left(2 \pi \nu \hat{z}-2 \tan ^{-1}(q \hat{z})+k s\right)}{1+(q \hat{z})^{2}} \\
& \times \exp \left[-\left(\frac{\hat{z}}{2 \hat{\sigma}_{\tau}}-\frac{s}{2 c \sigma_{\tau}}\right)^{2}\right],
\end{aligned}
$$

with $\hat{z}=c t / L_{u}, \nu=N 2 \delta \gamma / \gamma_{r}, q=L_{u} / z_{0}, \hat{\sigma}_{\tau}=\sigma_{\tau} / \tau_{0}$ and $\tau_{0}=2 \pi N / k c$. For the Bessel functions, the following expression is used:

$$
\{J J\}=J_{0}\left(\frac{\xi}{2}\right)-J_{1}\left(\frac{\xi}{2}\right) .
$$

The parameters of the laser are its pulse energy $A_{L}$, and its peak power defined by:

$$
P_{L}=\frac{A_{L}}{\sqrt{2 \pi} \sigma_{\tau}}=\frac{E_{0}^{2} \pi w_{0}^{2} c}{8 \pi}
$$

Then, Eq. (80) can be rewritten as:

$$
\begin{aligned}
\left\langle\frac{d \gamma}{d \hat{z}}\right\rangle= & k x_{0} q \frac{2 K}{\gamma} \sqrt{\frac{P_{L}}{P_{0}}}\{J J\} \frac{\cos \left(2 \pi \nu \hat{z}-2 \tan ^{-1}(q \hat{z})+k s\right)}{1+(q \hat{z})^{2}} \\
& \times \exp \left[-\left(\frac{\hat{z}}{2 \hat{\sigma}_{\tau}}-\frac{s}{2 c \sigma_{\tau}}\right)^{2}\right]
\end{aligned}
$$

where $P_{0}=I_{A} m c^{2} / e$, and $I_{A}$ the Alfvèn current. Using the Panofsky-Wenzel theorem and Eq. (81), the change of horizontal momentum of an electron in the undulator is given by:

$$
\begin{aligned}
\left\langle\frac{d x^{\prime}}{d \hat{z}}\right\rangle= & q \frac{2 K}{\gamma^{2}} \sqrt{\frac{P_{L}}{P_{0}}}\{J J\} \frac{\sin \left(2 \pi \nu \hat{z}-2 \tan ^{-1}(q \hat{z})+k s\right)}{1+(q \hat{z})^{2}} \\
& \times \exp \left[-\left(\frac{\hat{z}}{2 \hat{\sigma}_{\tau}}-\frac{s}{2 c \sigma_{\tau}}\right)^{2}\right],
\end{aligned}
$$


where $x^{\prime} \approx p_{x} / m c \gamma$. Eventually, the electron angular kick $\Delta x^{\prime}$, and the change of energy of the electron due to the interaction with the laser $\Delta \gamma$, are given by:

$$
\begin{aligned}
\frac{\Delta \gamma}{\gamma} & =\frac{2 K}{\gamma^{2}} \sqrt{\frac{P_{L}}{P_{0}}}\{J J\} k x_{0} f\left(q, \nu, \hat{\sigma}_{\tau}, s\right) \times \cos (k s+\varphi), \\
\Delta x^{\prime} & =\frac{2 K}{\gamma^{2}} \sqrt{\frac{P_{L}}{P_{0}}}\{J J\} f\left(q, \nu, \hat{\sigma}_{\tau}, s\right) \times \sin (k s+\varphi),
\end{aligned}
$$

where $f$ is defined by:

$$
f\left(q, \nu, \hat{\sigma}_{\tau}, s\right)=q \int_{0.5}^{0.5} \frac{\cos \left(2 \pi \nu \hat{z}-2 \tan ^{-1}(q \hat{z})\right)}{1+(q \hat{z})^{2}} \times \exp \left[-\left(\frac{\hat{z}}{2 \hat{\sigma}_{\tau}}-\frac{s}{2 c \sigma_{\tau}}\right)^{2}\right] .
$$




\section{B Echo-7 beamline}
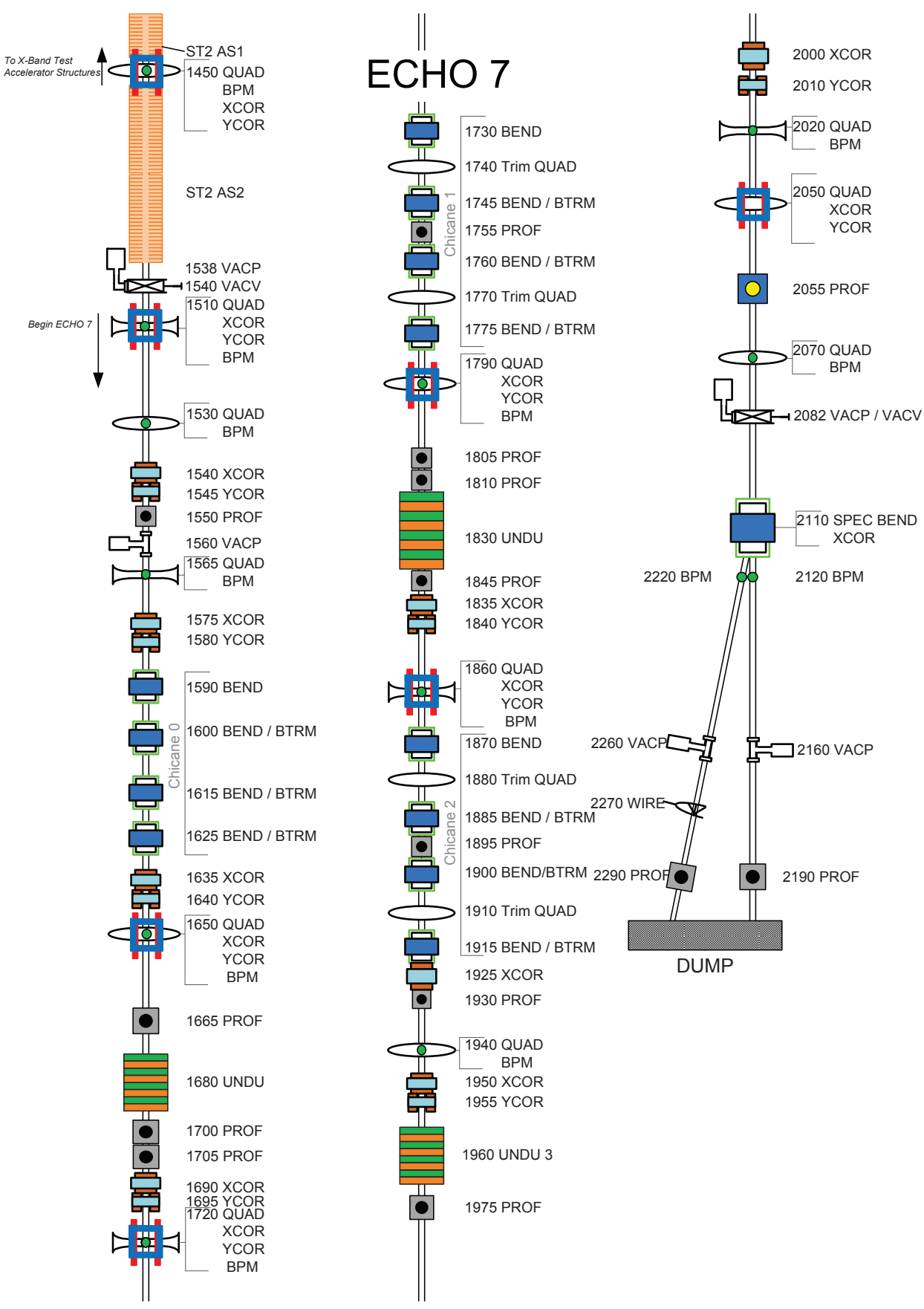

Figure 46: Beamline of the Echo-7 experiment at the NLCTA. 


\section{References}

[1] J. C. Maxwell, A treatise on electricity and magnetism, (Clarendon Press Series, 1873), Part. 4, Chap. 9.

[2] H. R. Hertz, Annalen der Physik 267, 421-448 (1887).

[3] A. Liénard, L’Éclairage Élec. 16, 5 (1898).

[4] E. Wiechert, Archives Néerlandaises 5, 549-573 (1900).

[5] G. A. Schott, Electromagnetic radiation, (Cambridge University Press, 1912).

[6] LBL website, http://xdb.1bl.gov/.

[7] PSI website, http://sls.web.psi.ch/.

[8] L. Rivkin, Introduction to particle accelerators course, (École Polytechnique Fédérale de Lausanne, Fall 2008).

[9] F. Pfeiffer, T. Weitkamp, O. Bunk, and C. David, Nature Physics 2, 258 - 261 (2006).

[10] D. Nguyen, S. Russell, and N. Moody, Theory and practice of free electron lasers, (US particle accelerator school, 2009).

[11] J. B. Murphy and C. Pellegrini, Introduction to the physics of the free electron laser, (Springer, 1988), Chap. 1.

[12] LCLS website, http://lcls.slac.stanford.edu/.

[13] S. Reiche, Production of seeded soft X-ray pulses, (PSI, February 26, 2009).

[14] G. Stupakov, Phys. Rev. Lett. 102, 074801 (2009).

[15] D. Xiang et al., Preliminary results of the echo-seeding experiment at SLAC, (IPAC 2010).

[16] Z. Huang, M. Borland, P. Emma, J. Wu, C. Limborg, G. Stupakov, and J. Welch, Phys. Rev. ST Accel. Beams 7, 074401 (2004).

[17] T. O. Raubenheimer, P. Emma, and S. Kheifets, Chicane and wiggler based bunch compressors for future linearcolliders, (IEEE Particle Accelerator Conference, 1994).

[18] D. Xiang and G. Stupakov, Phys. Rev. ST Accel. Beams 12, 030702 (2009).

[19] M. Abramovitz and I. Stegun, Handbook of mathematical functions.

[20] J. A. Clarke, The science and technology of undulators and wigglers, (Oxford University Press, 2004).

[21] G. Stupakov, private communication (05/03/2010, SLAC).

[22] A. A. Zholents and M. S. Zolotorev, New Journal of Physics 10, 025005 (2008).

[23] A. E. Sigman, Lasers University Science Books, (Mill Valley), 642. 